OPEN ACCESS

Edited by:

Touqeer Ahmed,

National University of Sciences \&

Technology, Pakistan

Reviewed by:

Budbazar Enkhjargal,

Boston University, United States

Baoliang Sun,

Shandong First Medical University,

China

*Correspondence:

Yan Qu

yanqu0123@fmmu.edu.cn

${ }^{\dagger}$ These authors have contributed equally to this work

Specialty section:

This article was submitted to

Neuropharmacology,

a section of the journal

Frontiers in Pharmacology

Received: 30 January 2020

Accepted: 30 April 2020

Published: 15 May 2020

Citation:

Liu H, Guo W, Guo H, Zhao L, Yue L

Li X, Feng D, Luo J, WU X, Cui $W$ and

Qu Y (2020) Bakuchiol Attenuates

Oxidative Stress and Neuron Damage

by Regulating TrX1/TXNIP and the

Phosphorylation of AMPK After Subarachnoid Hemorrhage in Mice.

Front. Pharmacol. 11:712.

doi: 10.3389/fphar.2020.00712

\section{Bakuchiol Attenuates Oxidative Stress and Neuron Damage by Regulating Trx1/TXNIP and the Phosphorylation of AMPK After Subarachnoid Hemorrhage in Mice}

\author{
Haixiao Liu ${ }^{1,2+}$, Wei Guo ${ }^{1+}$, Hao Guo ${ }^{1+}$, Lei Zhao ${ }^{1}$, Liang Yue ${ }^{1}$, Xia Li $^{1}$, Dayun Feng ${ }^{1}$, \\ Jianing Luo ${ }^{1}$, Xun $W^{1}{ }^{1}$, Wenxing Cui ${ }^{1}$ and Yan $Q u^{1 *}$ \\ ${ }^{1}$ Department of Neurosurgery, Tangdu Hospital, The Fourth Military Medical University, X'ian, China, ${ }^{2}$ Department of \\ Pathology, University of Texas Southwestern Medical Center, Dallas, TX, United States
}

Subarachnoid hemorrhage (SAH) is a fatal cerebrovascular condition with complex pathophysiology that reduces brain perfusion and causes cerebral functional impairments. An increasing number of studies indicate that early brain injury (EBI), which occurs within the first $72 \mathrm{~h}$ of $\mathrm{SAH}$, plays a crucial role in the poor prognosis of $\mathrm{SAH}$. Bakuchiol (Bak) has been demonstrated to have multiorgan protective effects owing to its antioxidative and anti-inflammatory properties. The present study was designed to investigate the effects of Bak on $\mathrm{EBI}$ after $\mathrm{SAH}$ and its underlying mechanisms. In this study, 428 adult male C57BL/6J mice weighing 20 to $25 \mathrm{~g}$ were observed to investigate the effects of Bak administration in an SAH animal model. The neurological function and brain edema were assessed. Content of MDA/3-NT/8-OHdG/superoxide anion and the activity of SOD and GSH-Px were tested. The function of the blood-brain barrier (BBB) and the protein levels of claudin-5, occludin, zonula occludens-1, and matrix metalloproteinase-9 were observed. TUNEL staining and Fluoro-Jade C staining were conducted to evaluate the death of neurons. Ultrastructural changes of the neurons were observed under the transmission electron microscope. Finally, the roles of Trx, TXNIP, and AMPK in the protective effect of Bak were investigated. The data showed that Bak administration 1) increased the survival rate and alleviated neurological functional deficits; 2) alleviated BBB disruption and brain edema; 3) attenuated oxidative stress by reducing reactive oxygen species, MDA, 3-NT, 8-OHdG, gp91 ${ }^{\text {phox }}$, and 4-HNE; increased the activities of SOD and GSH-Px; and alleviated the damage to the ultrastructure of mitochondria; 4) inhibited cellular apoptosis by regulating the protein levels of Bcl-2, Bax, and cleaved caspase-3; and 5) upregulated the protein levels of Trx1 as well as the phosphorylation of AMPK and downregulated the protein levels of TXNIP. Moreover, the protective effects of Bak were partially reversed by PX-12 and compound C. To summarize, Bak attenuates EBI after SAH by alleviating BBB disruption, oxidative 
stress, and apoptosis via regulating Trx1/TXNIP expression and the phosphorylation of AMPK. Its powerful protective effects might make Bak a promising novel drug for the treatment of $\mathrm{EBI}$ after $\mathrm{SAH}$.

Keywords: bakuchiol, subarachnoid hemorrhage, early brain injury, oxidative stress, apoptosis, thioredoxin, AMP-activated protein kinase

\section{INTRODUCTION}

Although the treatment of subarachnoid hemorrhage (SAH), a severe subtype of stroke, has been discussed a lot in recent years, the mortality and morbidity of SAH remain high and it leads to the loss of many years of productive life (Macdonald and Schweizer, 2017). The loss of neurological function results from the primary injury directly caused by the hemorrhage and secondary injury following the primary injury (Macdonald, 2014).

Recently, an increasing number of studies have indicated that early brain injury (EBI), which occurs within the first $72 \mathrm{~h}$ of $\mathrm{SAH}$, plays a crucial role in the poor prognosis of SAH (Liu L. et al., 2017; Macdonald and Schweizer, 2017). EBI is the primary cause of SAH-associated histological injuries, function deficits, and death (Sehba et al., 2012). Therefore, the targeting of EBI might be the most effective method for the treatment of SAH. During EBI, hemorrhage causes many pathophysiology problems, including the increase in intracranial pressure, the decrease in cerebral blood flow, and the global cerebral ischemia (Fujii et al., 2013). A complex mechanism, including blood-brain barrier (BBB) disruption, neuroinflammation, oxidative stress, and neuronal apoptosis is involved in the process of injury after $\mathrm{SAH}$, which ultimately leads to cell death and severe damage to neurological functions (Lucke-Wold et al., 2016).

Bakuchiol (Bak), [(1E,3S)-3-ethenyl-3,7-dimethyl-1,6-oct adien-1-yl] phenol, an analog of resveratrol, is a prenylated phenolic monoterpene isolated from the seeds of Psoralea corylifolia L. (Leguminosae) (Feng et al., 2016; Xin et al., 2019) Figure 1A. It was initially identified in Otholobium pubescens, a kind of Peruvian medicinal plant used for the treatment of diabetes (Krenisky et al., 1999). Recently, Bak has been demonstrated to have numerous pharmacological properties, including the antioxidative and anti-inflammatory, antidiabetic, antiaging, and anticancer properties (Choi et al., 2010; Seo et al., 2013; Chaudhuri and Bojanowski, 2014; Li L. et al., 2017; Lim et al., 2019; Xin et al., 2019). For example, Bak maintains the activities of mitochondrial respiratory enzyme and protects the functions of mitochondrial against oxidative stress injury (Haraguchi et al., 2000). Bak treatment could also alleviate the edema, inflammation, and oxidative stress in the sepsis-induced acute lung injury (Zhang et al., 2017). Besides, Bak treatment could attenuate myocardial ischemia-reperfusion injury by attenuating mitochondrial oxidative damage and apoptosis via the activation of the SIRT1/PGC-1 $\alpha$ signaling pathway (Feng et al., 2016). This strong antioxidative effect might be mediated by the terpenoid chain in its structure via a radical scavenging way (Adhikari et al., 2003). However, the effects of Bak on SAH remain unclear.

The present study aims to investigate the effects of Bak on EBI after SAH. The protective effects of Bak on BBB integrity, oxidative stress, cellular apoptosis, and neurological function during EBI were explored in an endovascular perforation $\mathrm{SAH}$ model in C57BL/6J mice. The roles of thioredoxin (Trx)/ thioredoxin-interacting protein (TXNIP) and AMP-activated protein kinase (AMPK), which are crucial for the regulation of molecules in intracellular oxidative stress, were then studied by using their selective inhibitor, PX-12 and compound C (CC).

\section{MATERIALS AND METHODS}

\section{Animals and Ethics}

Healthy adult male C57BL/6J mice weighing 20-25 g were obtained from the Animal Center of the Fourth Military Medical University. The mice were maintained on a $12 \mathrm{~h}$ light/ dark cycle at approximately $22^{\circ} \mathrm{C}$ under pathogen-free conditions with given free access to food and water. All experiments were performed according to The Guide for the Care and Use of Laboratory Animals published by the US National Institutes of Health (National Institutes of Health Publication, No. 85-23, revised 1996) and had been approved by the Ethics Committee of the Fourth Military Medical University (NO. TDLL2017-04-192).

\section{Reagents}

Bak, dihydroethidium (DHE), and 4',6-diamino-2-phenylindole (DAPI) were purchased from Sigma-Aldrich (St. Louis, MO, USA). 1-Methylpropyl 2-imidazolyl disulfide (PX-12) was purchased from Selleck Chem (Houston, TX, USA). CC (ab146597) and rabbit polyclonal antibodies against gp91 $1^{\text {phox }}$ (ab80508), 4-hydroxynonenal (4-HNE) (ab46545), cleaved caspase-3 (ab2302), claudin-5 (ab15106), occludin (ab216327), and zonula occludens-1 (ZO-1) (ab96587) were purchased from Abcam (Cambridge, UK). Rabbit monoclonal antibodies against B-cell lymphoma-2 (Bcl-2) (2870), Bcl-2-associated X protein (Bax) (14796), matrix metalloproteinase-9 (MMP-9) (13667S), Trx-1 (2429S), TXNIP (14715S), AMPK (2532S), and phosphoAMPK (Thr172) (D4D6D) were purchased from Cell Signaling Technology (Beverly, MA, USA). Rabbit polyclonal antibody against $\beta$-actin (AC006) was purchased from ABclonal Biotech (College Park, Maryland, USA). A terminal deoxynucleotidyl transferase uridine triphosphate (UTP) nick-end labeling (TUNEL) kit was purchased from Roche (Mannheim, 
Germany). Fluoro-Jade C (FJC) was purchased from Millipore (Temecula, USA). The enzyme-linked immune sorbent assay (ELISA) kits used to measure 8-hydroxy-2-deoxyguanosine (8$\mathrm{OHdG}$ ) and 3-nitrotyrosine (3-NT) levels were purchased from Cell Biolabs (San Diego, CA, USA). The kits used to measure glutathione peroxidase (GSH-Px), superoxide dismutase (SOD), and malondialdehyde (MDA) levels were purchased from the Institute of Jiancheng Bioengineering (Nanjing, Jiangsu, China). Human resource planning (HRP)-conjugated goat anti-rabbit secondary antibody was purchased from Bioworld Co. (Shanghai, China).

\section{Experimental Designs}

First, mice were randomly assigned to four groups: the sham, $\mathrm{SAH}, \mathrm{SAH}+$ vehicle, and SAH + Bak groups (Figure 1B). Sham or SAH operation was conducted in the appropriate groups. Bak was diluted to a concentration of $10 \mathrm{mg} / \mathrm{ml}$ in normal saline with a $2 \%$ volume of ethanol. Drugs were administered orally by gavage at a dosage of $50 \mathrm{mg} / \mathrm{kg} /$ day for seven consecutive days before the injury. The mice in the vehicle group were given equal doses of $2 \%$ ethanol dissolved in normal saline. Second, PX-12 (25 mg/kg), a selective Trx inhibitor, and CC (2 mg/kg), a selective AMPK inhibitor, were administered to mice in the $\mathrm{SAH}+\mathrm{Bak}+\mathrm{PX}-12$ and $\mathrm{SAH}+\mathrm{Bak}+\mathrm{CC}$ groups through tail vein injection immediately after SAH. The sham + Bak group was also introduced to observe the effects of Bak on normal animals. Tissues were collected 24 or $72 \mathrm{~h}$ after injury for the following experiments. All of the experiments and statistical analyses were conducted by researchers blinded to the grouping.

\section{Subarachnoid Hemorrhage Animal Model}

An endovascular perforation SAH model was developed by a method that has been described previously (Sozen et al., 2009; Kooijman et al., 2014). Briefly, animals were anesthetized with a mixture of isoflurane in 30\% oxygen and $70 \%$ nitrous oxide (3\% induction, $1.5 \%$ maintenance, $\mathrm{v} / \mathrm{v})$. Then, the left common carotid artery bifurcation was exposed, and the left external carotid artery was ligated and dissected. A nylon suture was inserted into the left internal carotid artery through the left external carotid artery stump and the left common carotid artery bifurcation. Resistance was encountered when the suture was located near the anterior communicating artery (ACA). The suture was then advanced approximately 3 more millimeters (total depth of $13-14 \mathrm{~mm}$ ) to perforate the ACA. The suture was maintained in this position for $30 \mathrm{~s}$ before its withdrawal. A similar operation was performed on mice in the sham group, except that the suture was pierced less than $8 \mathrm{~mm}$ to avoid perforation of the ACA. The temperature was continuously monitored during the surgery. The animals were kept warm using a heating plate to maintain body temperature at $36.5-37.5^{\circ} \mathrm{C}$ during surgery and within $2 \mathrm{~h}$ after surgery. Meloxicam was given as analgesic after surgery, and $5 \%$ glucose dissolved in normal saline was given as nutritional support. To ensure the uniformity of damage, the mice with an SAH grading score $\leq 7$ were excluded.

\section{Survival Rate and Subarachnoid Hemorrhage Grade}

Twenty mice in each group were used to evaluate the postinjury survival rate. These animals were observed for 7 days after sham or SAH operation under pathogen-free conditions with free access to food and water. They were then euthanized after 7 days.

$\mathrm{SAH}$ grading scores were evaluated when the animals were sacrificed using a previously reported method (Sugawara et al., 2008). Briefly, the basal cisterns of the animals were divided into six segments (Figure 1C) and the Willis circle and basilar arteries were observed. Subarachnoid blood clotting and the arteries on each segment were evaluated and scored from 0 to 3 score (grade 0: no subarachnoid blood; grade 1: minimal subarachnoid blood; grade 2: moderate blood clot with recognizable arteries; grade 3: blood clot obliterating all arteries within the segment). The SAH grading score was the sum of the scores of each segment.

\section{Neurological Score}

Neurological function was assessed using a modified Garcia's neurological scoring system (Garcia et al., 1995; Zhao et al., 2017) at 24 or $72 \mathrm{~h}$ after surgery. In brief, the evaluation consisted of six tests of the following: spontaneous activity $(0-3)$, symmetry in the movement of all four limbs (0-3), symmetry in the movement of forelimbs $(0-3)$, climbing $(0-3)$, response to trunk stimulation (0-3), and response to whisker stimulation (0-3). The maximum score was 18 ; the minimum score was 0 . Higher scores indicated better function.

\section{Brain Water Content}

The brain water content was tested by a previously described method (Xi et al., 2001; Jin et al., 2017) using the following formula: the brain water content $(\%)=[$ (wet weight - dry weight)/wet weight] $\times 100 \%$. The mice were deeply anesthetized and sacrificed 24 or $72 \mathrm{~h}$ after surgery. Their brains were removed 24 or $72 \mathrm{~h}$ after injury and divided into four parts: the left hemisphere, the right hemisphere, the cerebellum, and the brain stem. Then, the water contents of each part were tested separately. Tissues were weighed immediately to obtain the wet weight, followed by drying at 95 to $100^{\circ} \mathrm{C}$ for $72 \mathrm{~h}$ and then weighing to obtain the dry weight.

\section{Evans Blue Extravasation}

Evans blue (EB) extravasations were tested 24 and $72 \mathrm{~h}$ after injury using a spectrophotometer $(\lambda=610 \mathrm{~nm})$ to evaluate the permeability of the BBB. Briefly, $4 \mathrm{ml} / \mathrm{kg}$ of $2 \%$ (w/v) EB dye was injected into the right tail vein $3 \mathrm{~h}$ before the mice were sacrificed. Then, the animals were perfused transcardially with $50 \mathrm{ml}$ of ice-cold 0.1 M phosphate-buffered saline (PBS, pH 7.4) under anesthesia to remove intravascular EB dye. The ipsilateral cortex was removed and homogenized in PBS and an equal volume of trichloroacetic acid to precipitate the protein. After 5 min, the samples were centrifuged, and the supernatants were extracted and used to measure the absorbance (Zhang et al., 2014; Zhang et al., 2015a). 


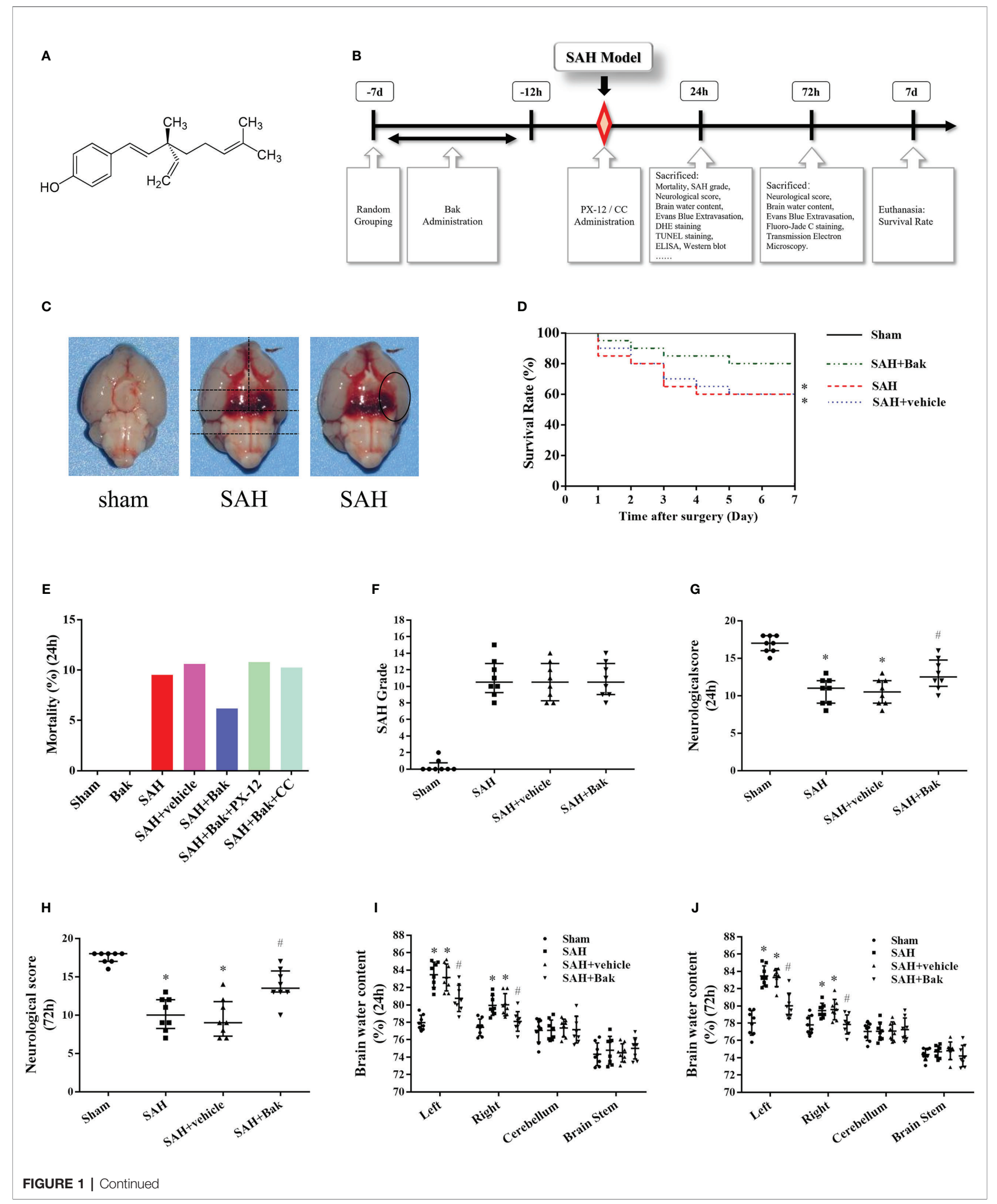


FIGURE 1 | Experimental protocol and effect of Bak on mortality, neurological score, and brain water content in each group. (A) The chemical structure of Bak. (B) Experimental protocol. (C) The brain after SAH or sham. Blood clots can be seen in the ventral brain after SAH. The method to evaluate the SAH grading scores and the area observed after staining were showed. (D) Effect of Bak on the 7-day survival rate after SAH. Survival percentages in each day after injury are shown. Values are expressed as survival percentage. $n=20$ for each group. (E) The mortality in each group. (F) SAH grading scores in each group. $n=8$ for each group. (G, H) The neurological scores at 24 and $72 \mathrm{~h}$ after SAH. n=8 for each group. (I, J) Brain water content at 24 and $72 \mathrm{~h}$ after SAH. The brains are divided into four parts: the left hemisphere, the right hemisphere, the cerebellum, and the brain stem. The water content of each part is shown separately. $n=8$ for each group. Values of SAH grading score and neurological score are expressed as median and 25 th-75th percentiles. Other values are expressed as mean \pm SD. ${ }^{\star} P<0.05$ vs. sham group, ${ }^{\#} P<0.05$ vs. SAH + vehicle group. Bak, bakuchiol; SAH, subarachnoid hemorrhage.

\section{Dihydroethidium Staining}

DHE staining was conducted to detect the superoxide anion, which reflected the oxidant stress levels in the tissue. Animals were perfused transcardially with PBS under anesthesia $24 \mathrm{~h}$ after $\mathrm{SAH}$. Samples were immediately frozen at $-80^{\circ} \mathrm{C}$ and sliced into $15 \mu \mathrm{m}$ thick coronal brain slice with a freezing microtome (CM 1950, Leica, German). With reference to The Mouse Brain in Stereotaxic Coordinates (Second Edition) (ACADEMIC PRESS), the slices at $0.58-2.5 \mathrm{~mm}$ posterior to bregma were selected. The slices were dyed with DHE for $30 \mathrm{~min}$. Then, the ventral side of the left hemisphere was observed with a laser scanning confocal microscope (A1 Si, Nikon, Japan) (Figure 1C). The representative images were obtained from the slices located at about $2 \mathrm{~mm}$ posterior to bregma.

\section{Assay of Malondialdehyde Content and the Superoxide Dismutase and Glutathione Peroxidase Activities}

The brains were removed after their perfusion with PBS $24 \mathrm{~h}$ after SAH, and the ipsilateral cortex was homogenized to detect the levels of MDA and the activities of oxidative stress-related enzymes (SOD and GSH-Px). According to the instructions of commercial kits, the MDA levels were tested by the reaction of MDA with thiobarbituric acid under acidic conditions and a high temperature, following which the absorbance was detected. SOD activity was tested by WST-1 method following the instruction of commercial kits. The GSH-Px activity was tested by detecting the reduction of NADPH in the reaction system following the instructions of a commercial kit.

\section{Concentrations of 3-Nitrotyrosine and 8-Hydroxy-2-Deoxyguanosine}

ELISA kits were used to evaluate the levels of 3-NT and 8-OHdG in injured tissues at $24 \mathrm{~h}$ after SAH. Briefly, samples or standards were incubated with primary antibody at $4^{\circ} \mathrm{C}$ overnight, secondary antibody at room temperature for $1 \mathrm{~h}$, and a substrate solution at the room temperature for $15 \mathrm{~min}$ in the dark. Finally, a solution to terminate the reaction was added to each sample. A SpectraMax M2 spectrometer (Molecular Devices, Sunnyvale, CA, USA) was used to measure the absorbance and calculate the protein level.

\section{Transmission Electron Microscopic Observation}

Samples were prepared following previously reported methods (Li $\mathrm{X}$. et al., 2017). Briefly, $72 \mathrm{~h}$ after SAH induction, the mice were anesthetized and perfused with $50 \mathrm{ml}$ of ice-cold PBS and $60 \mathrm{ml}$ of ice-cold $4 \%$ paraformaldehyde (PFA). Then, the brains were removed. The injured cortical tissues were cut perpendicular to the long axis and trimmed into $1.5 \mathrm{~mm} \times 1.5 \mathrm{~mm} \times 3 \mathrm{~mm}$ blocks. Then, the specimens were fixed for $12 \mathrm{~h}$ in $4 \%$ glutaraldehyde, postfixed for $1 \mathrm{~h}$ in $1 \%$ osmium tetroxide, dehydrated through graded ethanol, and embedded in resin. Specimens were cut into $80 \mathrm{~nm}$ sections by an ultramicrotome (Leica, Vienna, Austria). The ultrathin sections were fixed on 200 slot grids coated with Pioloform membranes and observed with a JEM-1400 electron microscope (JEOL, Tokyo, Japan). Micrographs were captured with a charge-coupled device camera (Olympus, Tokyo, Japan).

\section{Terminal Deoxynucleotidyl Transferase Uridine Triphosphate Nick-End Labeling Assay}

A TUNEL kit was used to detect cell apoptosis in the injured cortex. The animals were perfused with PBS and PFA $24 \mathrm{~h}$ after the injury as described above. Their brains were cautiously removed, fixed in $4 \%$ PFA for $12 \mathrm{~h}$ and dehydrated in sucrose solutions at different concentrations $(10,20$, and $30 \%)$. The tissues were sliced into $25 \mu \mathrm{m}$ thick slices with a freezing microtome. The slices were incubated with $0.3 \%$ hydrogen peroxide for $30 \mathrm{~min}$ at room temperature, $0.25 \%$ pancreatin for $45 \mathrm{~min}$ at $37^{\circ} \mathrm{C}$, TUNEL reaction solution for $60 \mathrm{~min}$ at $37^{\circ} \mathrm{C}$ and DAPI $(5 \mu \mathrm{g} / \mathrm{ml})$ staining solution for $10 \mathrm{~min}$ at $37^{\circ} \mathrm{C}$ in a humidified box in the dark. With reference to The Mouse Brain in Stereotaxic Coordinates (Second Edition) (ACADEMIC PRESS), the slices at $0.58-2.5 \mathrm{~mm}$ posterior to bregma were selected. The ventral side of the left hemisphere was observed with a confocal microscope (Figure 1C). The representative images were obtained from the slices located at about $2 \mathrm{~mm}$ posterior to bregma. The apoptotic index was reflected by the ratio of TUNEL-positive cells to DAPI-positive cells.

\section{Fluoro-Jade C Staining}

FJC staining was performed to detect neuronal degeneration in the tissues $72 \mathrm{~h}$ after injury (Bai et al., 2018). Briefly, the tissues were perfused, collected, fixed, dehydrated, and sliced as described above. The observed slices and areas were selected according to the method described above. Selected sections were incubated with $1 \% \mathrm{NaOH}$ in $80 \%$ ethanol for $5 \mathrm{~min}$ and then rehydrated with $70 \%$ ethanol for $2 \mathrm{~min}$ and distilled water for 2 $\mathrm{min}$. The slices were then incubated with $0.06 \% \mathrm{KMnO}_{4}$ for 10 $\mathrm{min}$, rinsed with distilled water for $3 \mathrm{~min}$, and incubated with a $0.0001 \%$ FJC solution for $15 \mathrm{~min}$. Finally, the slices were washed three times with distilled water for $1 \mathrm{~min}$ each. The ventral side of the left hemisphere was observed and images were obtained 
using a confocal microscope (Figure 1C). FJC-positive neurons were counted and calculated.

\section{Western Blot Analysis}

The ipsilateral cortical samples collected $24 \mathrm{~h}$ after injury were sonicated and homogenized in a mixture of lysis buffer and $1 \%$ protease inhibitor for $30 \mathrm{~min}$ and then centrifuged for $15 \mathrm{~min}$ at $12,000 \mathrm{rpm}$. Equal amounts of protein $(25 \mu \mathrm{g})$ were separated on 8-15\% sodium dodecyl sulfate (SDS)-polyacrylamide gels and transferred onto polyvinylidene difluoride (PVDF) membranes (Millipore Corporation, USA). The PVDF membranes were blocked in 5\% nonfat dry milk/TBST (Tris-buffered saline, $0.1 \%$ Tween 20) for $90 \mathrm{~min}$ at room temperature and then incubated with rabbit anti-claudin-5 (1:1,000), anti-occludin $(1: 1,000)$, anti-ZO-1 (1:1,000), anti-MMP-9 (1:1,000), antigp91phox (1:500), anti-4-HNE (1:500), anti-Bcl-2 (1:1,000), anti-Bax (1:1,000), anti-cleaved caspase-3 (1:1,000), anti-Trx 1 $(1: 1,000)$, anti-TXNIP $(1: 1,000)$, anti-AMPK $(1: 1,000)$, antiphospho-AMPK $(1: 1,000)$, and anti- $\beta$-actin $(1: 1,000)$ primary antibodies overnight at $4^{\circ} \mathrm{C}$, followed by HRP-conjugated goat anti-rabbit $(1: 5,000)$ secondary antibody for $90 \mathrm{~min}$ at room temperature. Finally, the membranes were detected with the BioRad imaging system (Bio-Rad, Hercules, CA, USA).

\section{Statistical Analysis}

GraphPad Prism 6 (GraphPad Software, San Diego, CA, USA) and SPSS 18.0 (SPSS, Chicago, IL, USA) was used for analysis. The survival rate was analyzed with the log-rank (Mantel-Cox) test. $\mathrm{SAH}$ grading scores and neurological scores are expressed as medians and 25th-75th percentiles and were analyzed by the Kruskal-Wallis one-way analysis of variance (ANOVA) on ranks, followed by Tukey's post hoc analysis. Means \pm SDs are provided to describe other data. One-way ANOVA, followed by Tukey's post hoc analysis, and Bonferroni multiple comparison tests were used for intergroup comparisons. Multiple group comparisons were tested by one-way ANOVA followed by Tukey's honest significant difference (HSD) post hoc test. Differences for which $P$ $<0.05$ were considered statistically significant.

\section{RESULTS}

\section{Bak Increases the Survival Rate and Alleviates Neurological Functional Deficits and Brain Edema After Subarachnoid Hemorrhage}

The numbers of animals in each group used in the present study were described in Table 1. The overall mortality rate within $24 \mathrm{~h}$ after surgery in the sham, Bak, SAH, SAH + vehicle, $\mathrm{SAH}+\mathrm{Bak}$, $\mathrm{SAH}+\mathrm{Bak}+\mathrm{PX}-12$, and $\mathrm{SAH}+\mathrm{Bak}+\mathrm{CC}$ groups were $0 \%(0 /$ 92), $0 \%$ (0/6), 9.5\% (10/105), 10.6\% (12/113), 6.2\% (7/113), $10.8 \%(4 / 37)$, and $10.3 \%(4 / 39)$, respectively (Figure 1E). No animal was excluded from the experiment to observe the 7-day survival rate. The survived animals with insufficient brain injury were excluded from other experiments. Finally, 10, 9, 14, 3, and 4 animals were excluded in the $\mathrm{SAH}, \mathrm{SAH}+$ vehicle, $\mathrm{SAH}+\mathrm{Bak}$, $\mathrm{SAH}+\mathrm{Bak}+\mathrm{PX}-12$, and $\mathrm{SAH}+\mathrm{Bak}+\mathrm{CC}$ groups, respectively; 9, 12, 7, 4, 5 animals died before being sacrificed in the $\mathrm{SAH}$, $\mathrm{SAH}+$ vehicle, $\mathrm{SAH}+\mathrm{Bak}, \mathrm{SAH}+\mathrm{Bak}+\mathrm{PX}-12$, and $\mathrm{SAH}+$ Bak + CC groups, respectively (Table $\mathbf{1}$ ).

The 7-day survival rates in the sham, SAH, SAH + vehicle, and SAH + Bak groups were 100,60,60, and 80\%, respectively (Figure 1D). Blood clots were clearly visible around the Willis circle and ventral brainstem of animals in the SAH, SAH + vehicle, and $\mathrm{SAH}+\mathrm{Bak}$ groups. There was no significant difference in SAH grading scores between the SAH, SAH + vehicle, and SAH + Bak groups (Figure 1F).

Functional deficits, which were evaluated by neurological scores, and the degree of brain edema, which was evaluated by determining the brain water content, were measured 24 and $72 \mathrm{~h}$ after injury. SAH caused apparent neurological deficits and brain

TABLE 1 | The numbers of animals in each group.

\begin{tabular}{|c|c|c|c|c|c|c|c|}
\hline & \multirow[t]{2}{*}{ sham } & \multirow[t]{2}{*}{ sham+Bak } & \multirow[t]{2}{*}{ SAH } & \multicolumn{4}{|c|}{ SAH } \\
\hline & & & & Vehicle & Bak & Bak+PX-12 & Bak+CC \\
\hline \multicolumn{8}{|l|}{ Included } \\
\hline Survival rate & 20 & 0 & 20 & 20 & 20 & 0 & 0 \\
\hline Brain water content & 16 & 0 & 16 & 16 & 16 & 8 & 8 \\
\hline Evans blue extravasation & 14 & 0 & 14 & 14 & 14 & 7 & 7 \\
\hline DHE staining & 6 & 0 & 6 & 6 & 6 & 0 & 0 \\
\hline Oxidative stress marker detection & 6 & 0 & 6 & 6 & 6 & 6 & 6 \\
\hline Western Blot & 12 & 6 & 6 & 12 & 12 & 6 & 6 \\
\hline TUNEL & 6 & 0 & 6 & 6 & 6 & 0 & 0 \\
\hline FJC staining & 6 & 0 & 6 & 6 & 6 & 0 & 0 \\
\hline Transmission electron microscopy & 6 & 0 & 6 & 6 & 6 & 3 & 3 \\
\hline In total & 92 & 6 & 86 & 92 & 92 & 30 & 30 \\
\hline Died before being sacrificed & 0 & 0 & 9 & 12 & 7 & 4 & 5 \\
\hline Excluded* & 0 & 0 & 10 & 9 & 14 & 3 & 4 \\
\hline
\end{tabular}

*No animal was excluded from the experiment to observe the survival rate. The animal with a SAH grading score $\leq 7$ were excluded in other experiments. Bak, bakuchiol; SAH, subarachnoid hemorrhage; PX-12, 1-methylpropyl 2-imidazolyl disulfide; CC, compound C; DHE, dihydroethidium; TUNEL, terminal deoxynucleotidyl transferase UTP nick-end labeling; FJC, Fluoro-Jade C. 
edema. There were no significant differences in neurological scores or brain water content between the SAH and SAH + vehicle groups. Bak significantly improved neurological deficits and decreased the brain water content at 24 and $72 \mathrm{~h}$ after SAH (Figures 1G-J). The data of $24 \mathrm{~h}$-brain water content/ neurological scores and $72 \mathrm{~h}$-brain water content/neurological scores were collected from different batches of animals. Thus, the difference between the injuries at 24 and $72 \mathrm{~h}$ was not compared.

\section{Bak Protects Blood-Brain Barrier Integrity After Subarachnoid Hemorrhage}

EB extravasation was tested 24 and $72 \mathrm{~h}$ after injury to evaluate the permeability of BBB. A sharp increase in EB extravasation was observed in the SAH and SAH + vehicle groups ( $v s$. the sham group, $P<0.05$ ), which was alleviated by Bak (Figure 2B). In addition, decreases in levels of the tight junction proteins claudin-5, occludin, and ZO-1 were observed in the SAH and $\mathrm{SAH}+$ vehicle groups ( $v s$. the sham group, $P<0.05) 24 \mathrm{~h}$ after injury, which were offset by Bak. The MMP-9 level was increased in the SAH group ( $v s$. the sham group, $P<0.05$ ), but significantly reduced by Bak (Figures $\mathbf{2 A}, \mathbf{C}-\mathbf{F}$ ).

\section{Bak Ameliorates Oxidative Stress After Subarachnoid Hemorrhage}

The proportion of DHE-positive cells was dramatically increased after SAH and significantly decreased by Bak (Figures 3A, C).
The levels of MDA were significantly increased after SAH but significantly decreased following Bak administration (Figure 3D). In addition, the SOD and GSH-Px activities were impaired by $\mathrm{SAH}$ and remarkably enhanced by Bak administration (Figures 3E, F). Moreover, the levels of 3-NT and $8-\mathrm{OHdG}$ were also increased after $\mathrm{SAH}$ and decreased significantly by Bak administration (Figures 3G, H). The protein levels of gp91 ${ }^{\text {phox }}$ and 4 -HNE in the injured cortices were also increased $24 \mathrm{~h}$ after SAH and reduced by Bak treatment (Figures 3B, I, J).

\section{Bak Attenuates Neuronal Damage After Subarachnoid Hemorrhage}

TUNEL staining was performed to observe cellular apoptosis. The proportion of apoptotic cells was significantly increased after SAH. Bak significantly decreased the apoptotic index (Figures 4A, B). Previously, it was believed that the apoptosis mainly occurred on neurons after stroke. However, the latest research showed that the apoptosis and cell loss also occurred on glial cells (Chen et al., 2017; Ma et al., 2017; Sekerdag et al., 2018). Thus the FJC staining was further performed to quantify the degenerated neurons. The number of FJC-positive degenerated cells was significantly increased after SAH. This degeneration was alleviated by Bak administration (Figures 4C, D).

The protein levels of Bax, Bcl-2, and cleaved caspase- 3 in injured tissues were tested by western blotting. The protein levels
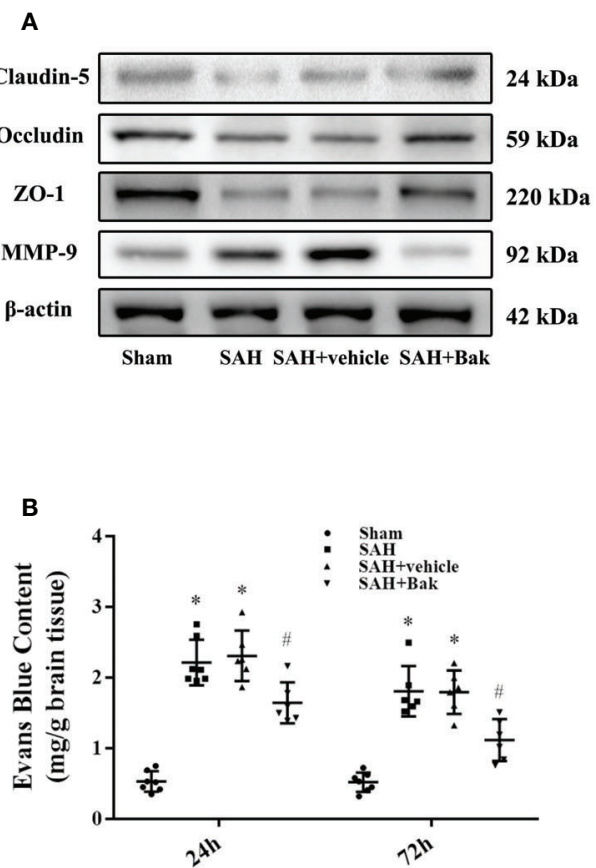
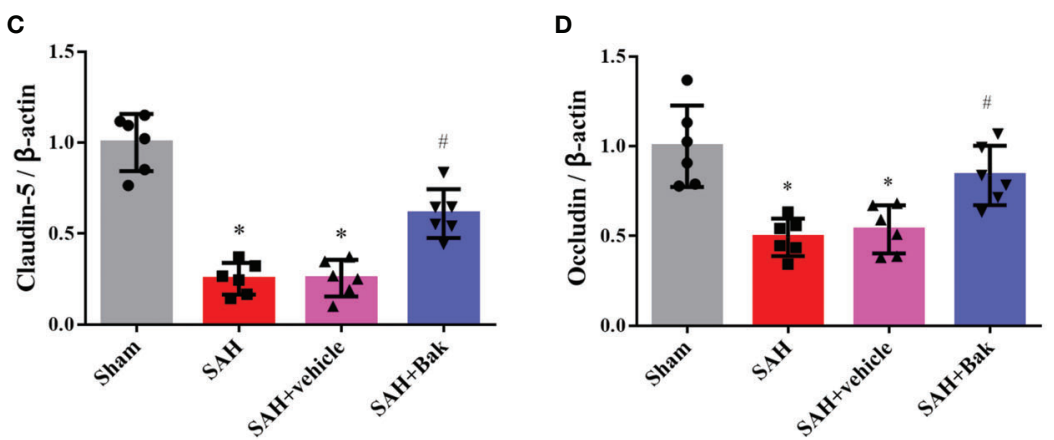

E

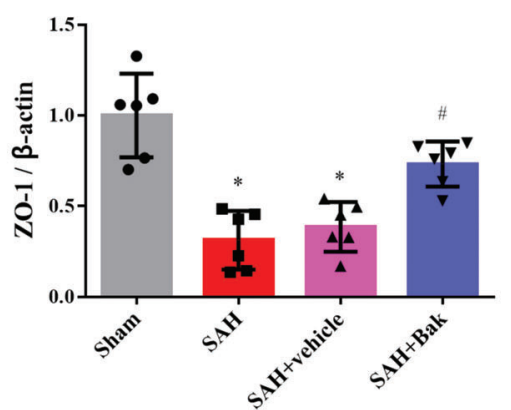

$\mathbf{F}$

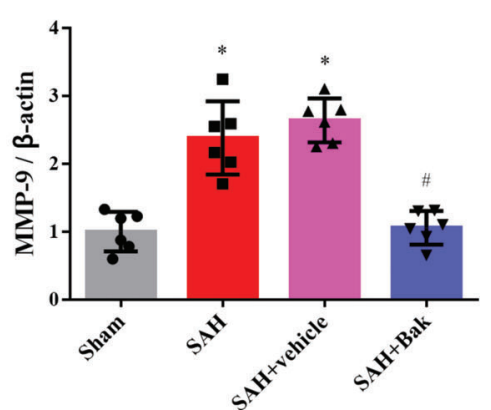

FIGURE 2 | The effect of Bak on BBB integrity after SAH. (A) Representative western blot images of the level of claudin-5, occludin, ZO-1, and MMP-9 at 24 h after $\mathrm{SAH} . \mathrm{n}=6$ for each group. (B) The EB extravasations at 24 and $72 \mathrm{~h}$ after injury. $\mathrm{n}=7$ for each group. (C-F) Statistical analysis of the protein level of claudin-5, occludin, ZO-1, and MMP-9. Values are expressed as mean $\pm \mathrm{SD}$. ${ }^{\star} P<0.05$ vs. sham group, ${ }^{\#} P<0.05$ vs. SAH + vehicle group. Bak, bakuchiol; SAH, subarachnoid hemorrhage; BBB, blood-brain barrier; ZO-1, zonula occludens-1; MMP-9, matrix metalloproteinase-9; EB, Evans blue. 


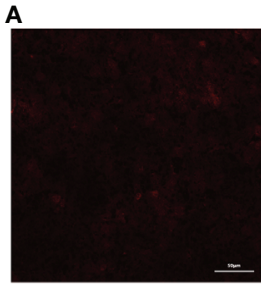

Sham

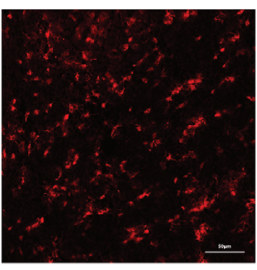

SAH

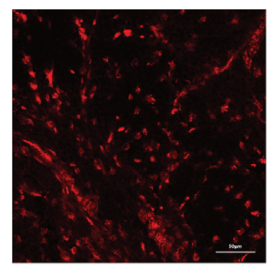

SAH+vehicle

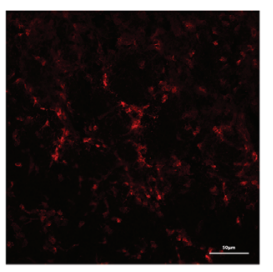

SAH+Bak

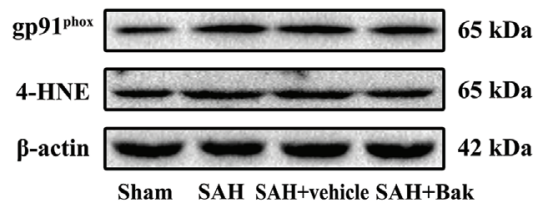

$\mathbf{F}$
C

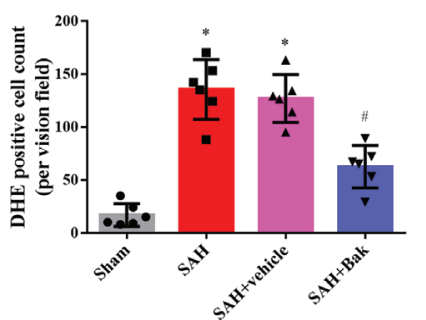

G

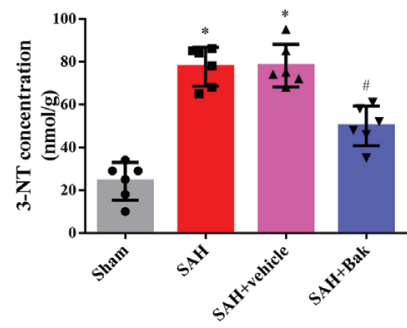

D

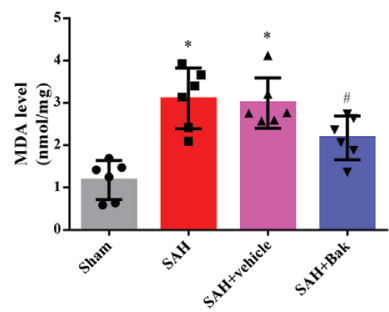

H

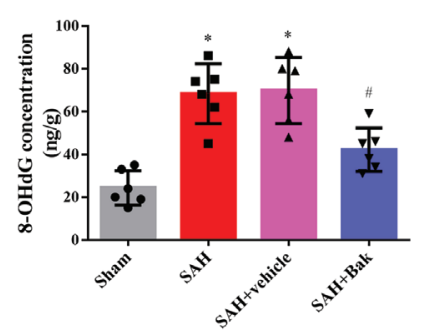

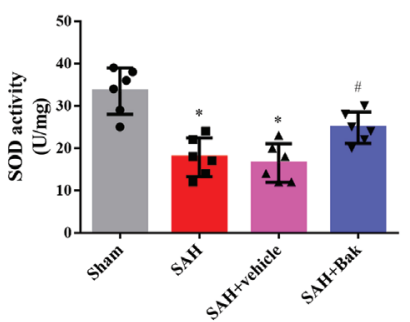

I

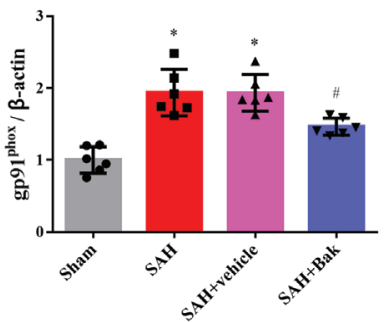

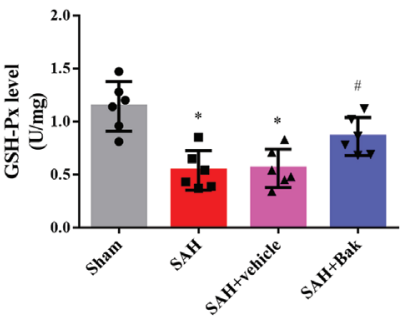

J

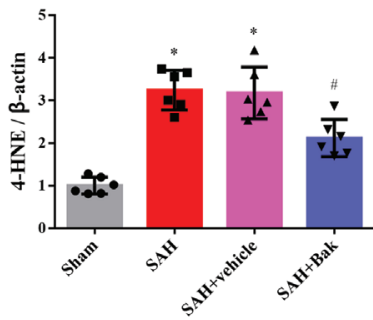

FIGURE 3 | The effect of Bak on the oxidative stress $24 \mathrm{~h}$ after SAH. (A, C) Representative images and quantitative analyses of DHE staining. Scale bar = 50 um. (D-F) The effect of Bak on the level of MDA, and on the activity of SOD and GSH-Px after SAH. (G, H) The effect of Bak on 8-OHdG and 3-NT concentrations after $\mathrm{SAH}$. (B, I, J) The effects of Bak on the gp91 ${ }^{\text {phox }}$ and 4-hydroxynonenal (4-HNE) after SAH. The representative western blot images and statistical analysis of the protein levels of gp9 $1^{\text {phox }}$ and 4 -HNE are shown. Values are expressed as mean $\pm \mathrm{SD}, \mathrm{n}=6$ for each group. ${ }^{\star} P<0.05$ vs. sham group, ${ }^{\#} P<0.05$ vs. SAH + vehicle group. Bak, bakuchiol; SAH, subarachnoid hemorrhage; DHE, dihydroethidium; MDA, malondialdehyde; SOD, superoxide dismutase; GSH-Px, glutathione peroxidase; 8-OHdG, 8-hydroxy-2-deoxyguanosine; 3-NT, 3-nitrotyrosine.

of Bax and cleaved caspase-3 were increased $24 \mathrm{~h}$ after SAH, which were partially offset by Bak. However, the protein level of Bcl-2 was decreased $24 \mathrm{~h}$ after $\mathrm{SAH}$, which was significantly ameliorated by Bak (Figures 4E-H).

\section{The Role of Trx/TXNIP and AMPK in the Protective Effects of Bak Against Subarachnoid Hemorrhage}

Western blotting was conducted $24 \mathrm{~h}$ after SAH to explore the role of Trx, TXNIP, and AMPK in the protective effects of Bak. The protein level of Trx was significantly decreased after SAH and increased after Bak administration. In contrast, the protein level of TXNIP was significantly increased after injury and decreased after Bak administration. In addition, the phosphorylation of AMPK was significantly increased after SAH. Bak administration further increased the level of phosphorylated AMPK (vs. the SAH + vehicle group, $P<0.05$ ) Besides, an increase of AMPK phosphorylation also observed in the sham + Bak group ( $v s$. the sham group, $P<0.05$ ) (Figures 5A-D).

Then, PX-12, a Trx inhibitor, and CC, an AMPK inhibitor, were used as negative controls to further explore the roles of AMPK and Trx/TXNIP in this mechanism. The neurological score was decreased, and the brain water content and EB extravasation were obviously increased after the administration of PX-12 or CC compared to those in the SAH + Bak group (Figures 5E-G). The MDA level was significantly increased by PX-12 and CC (vs. the $\mathrm{SAH}+$ Bak group, $P<0.05)$. In contrast, the activities of GSH-Px and SOD were downregulated by PX-12 and CC (vs. the SAH + Bak group, $P<0.05$ ) (Figures 5H-J). Meanwhile, transmission electron micrographs were used to observe ultrastructural changes in the mitochondria of neurons. Neurons in the SAH + Bak + PX12 and $\mathrm{SAH}+\mathrm{Bak}+\mathrm{CC}$ groups were characterized by the loss of mitochondrial cristae, swollen mitochondria, and morphological changes in the endoplasmic reticulum, which was similar to neurons in the SAH group (Figure 5K). 


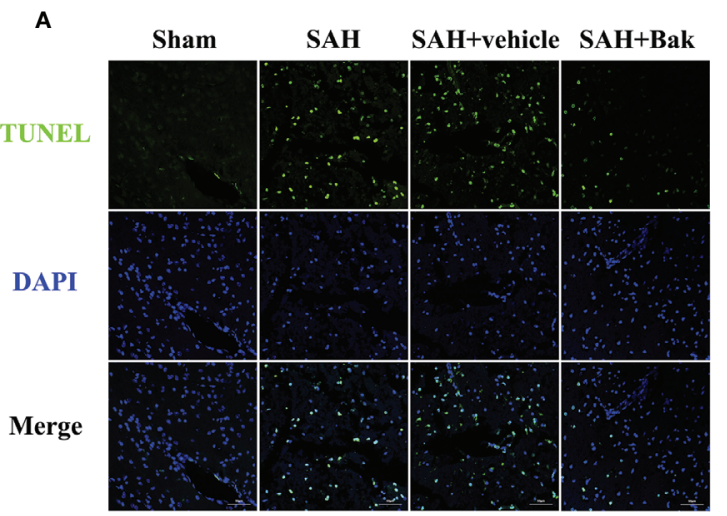

B

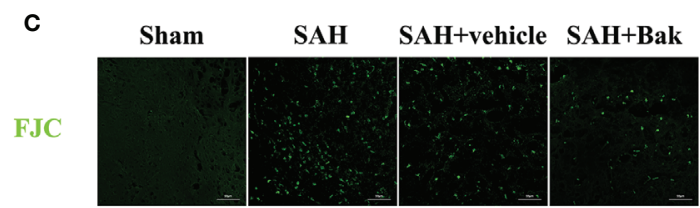

D

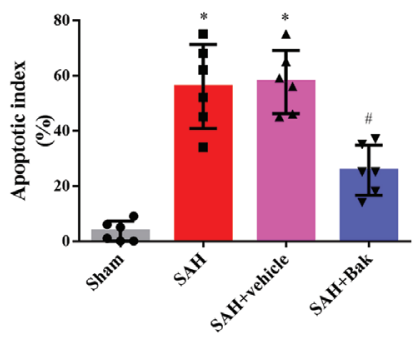

DAPI
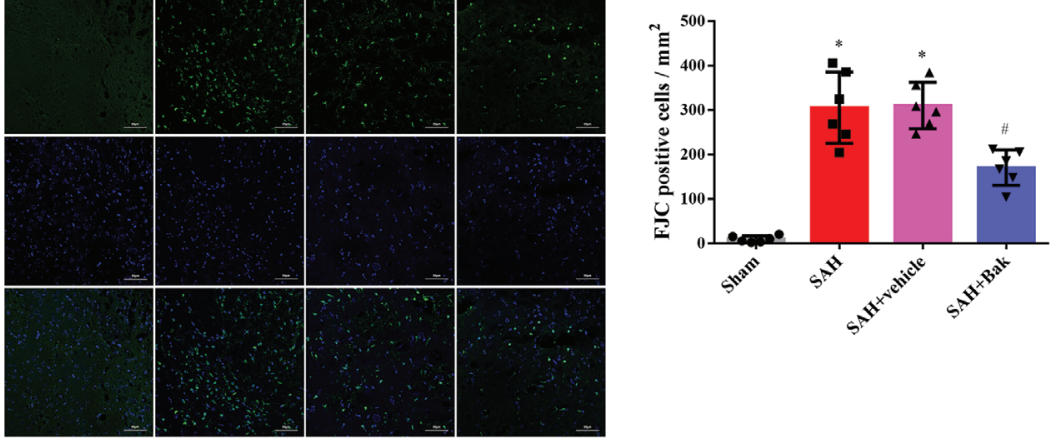

Merge

E

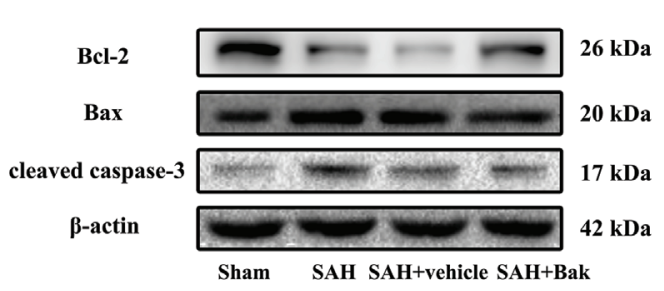

G

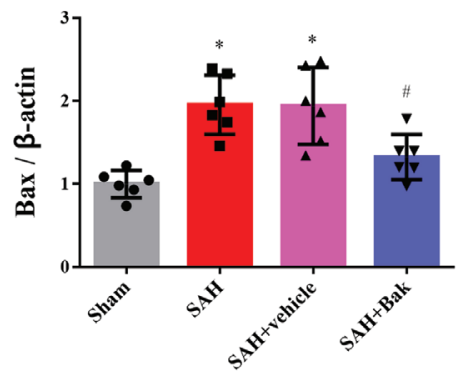

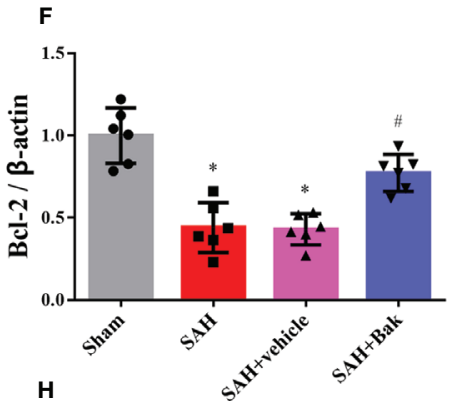

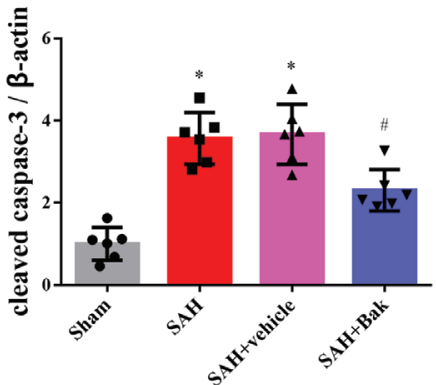

FIGURE 4 | The effects of Bak on cellular apoptosis and neuronal degeneration following SAH. (A, B) Representative images of TUNEL staining and quantitative analyses of TUNEL positive cells. Scale bar $=50 \mu \mathrm{m}$. (C, D) Representative images of FJC staining and quantitative analyses of FJC positive cells. Scale bar $=50$ $\mu \mathrm{m}$. (E-H) The effects of Bak on the apoptosis signaling $24 \mathrm{~h}$ after SAH. The representative western blot images and statistical analysis of the protein levels of Bcl-2, Bax, and cleaved caspase-3 are shown. Values are expressed as mean $\pm \mathrm{SD}, \mathrm{n}=6$ for each group. ${ }^{*} P<0.05$ vs. sham group, ${ }^{\#} P<0.05$ vs. SAH + vehicle group. Bak, bakuchiol; SAH, subarachnoid hemorrhage; TUNEL, terminal deoxynucleotidyl transferase UTP nick-end labeling; FJC, Fluoro-Jade C. 


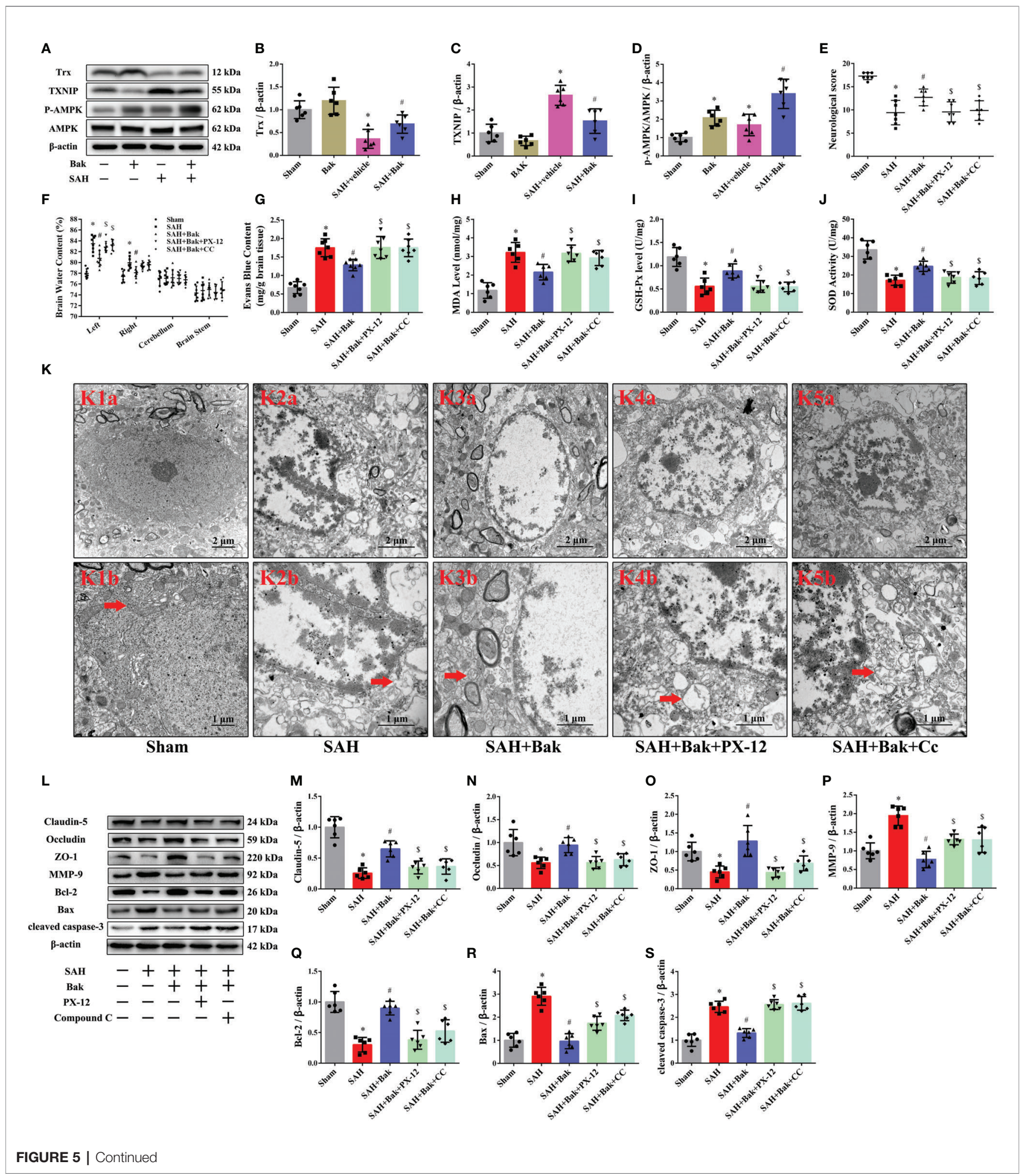


FIGURE 5 | The role of Trx/TXNIP and AMPK in the protective effect of Bak after SAH. (A-D) The representative western blot images and statistical analysis of the protein levels of Trx and TXNIP and the phosphorylation of AMPK at $24 \mathrm{~h}$ after SAH. $\mathrm{n}=6$ for each group. The protein levels of Trx and the phosphorylation levels of AMPK are up-regulated significantly by Bak (vs. the SAH + vehicle group). Then, the PX-12 and CC were used to identify the role of Trx and AMPK in the protective mechanism of Bak. (E) The neurological scores at 24 h. $n=7$ for each group. Values are expressed as median and 25th-75th percentiles. (F) Brain water content at 24 h. $n=8$ for each group. (G) Evans blue (EB) extravasation at 24 h. $n=7$ for each group. (H-J) The level of MDA, and the activity of SOD and GSH-Px in each group. $n=6$ for each group. (K) The ultrastructure of neurons in each group. K1b-K4b are the enlargements representative ultrastructure of neurons in K1a-K4a respectively. Scale bar $=2 \mu \mathrm{m}$ in $\mathrm{K} 1 \mathrm{a}-\mathrm{K} 4 \mathrm{a}, 1 \mu \mathrm{m}$ in K1b-K4b. Arrows indicate the mitochondria. (L-S) The representative western blot images and statistical analysis of the protein levels of claudin-5, occludin, ZO-1, MMP-9, Bcl-2, Bax, and cleaved caspase-3 at $24 \mathrm{~h}$. $\mathrm{n}=6$ for each group. Values are expressed as mean \pm SD except the neurological score, ${ }^{\star} P<0.05$ vs. sham group, ${ }^{\#} P<0.05$ vs. SAH + vehicle group, ${ }^{\$} P<0.05$ vs. SAH + Bak group. Bak, bakuchiol; SAH,

subarachnoid hemorrhage; Trx, thioredoxin; TXNIP, thioredoxin-interacting protein; AMPK, AMP-activated protein kinase; PX-12, 1-methylpropyl 2-imidazolyl disulfide; CC, compound C; MDA, malondialdehyde; SOD, superoxide dismutase; GSH-Px, glutathione peroxidase; ZO-1, zonula occludens-1; MMP-9, matrix metalloproteinase-9.

BBB integrity and cellular apoptosis were also tested $24 \mathrm{~h}$ after injury. The increased protein levels of claudin-5, occludin, ZO-1, and $\mathrm{Bcl}-2$ after Bak administration were significantly reduced by PX-12 or CC. In addition, the levels of MMP-9 and Bax were increased significantly by PX-12 or CC (Figures 5L-S).

\section{DISCUSSION}

SAH accounts for $5 \%$ of all strokes and occurs at a fairly young age (van Gijn et al., 2007). Patients with SAH often have cognitive impairments, which severely affect their ability to work and quality of life (Macdonald and Schweizer, 2017). However, the current SAH treatment strategy does not achieve a satisfactory functional outcome. Thus, the need to find better treatment is urgent.

EBI is the most important cause of disability and death after SAH. The treatment of EBI may successfully attenuate some of the devastating secondary injuries and improve the outcome of SAH patients 5 . Thus, attenuating EBI is the main goal of SAH treatment and a crucial method to reduce disability and mortality (Cahill and Zhang, 2009).

Bak, a prenylated phenolic monoterpene, is used in both traditional Chinese medicine and traditional Indian medicine (Chen et al., 2010). Recently, Bak was demonstrated to have multiorgan protective effects through a variety of pharmacological activities (Xin et al., 2019). In the present study, oral administration of Bak 1) reduced the mortality rate and improved the neurological function of animals after SAH, 2) attenuated disruption to the BBB and brain edema caused by $\mathrm{SAH}, 3)$ reduced the superoxide production, alleviated oxidative stress, and protected the mitochondrial ultrastructure during EBI, 4) attenuated SAH-induced cellular apoptosis and neuron damage, and 5) regulated the protein levels of Trx and TXNIP and the phosphorylation of AMPK. Moreover, both PX-12, a selective Trx inhibitor (Ji et al., 2019), and CC, a selective AMPK inhibitor (Oliveira et al., 2012; Guo et al., 2018), reversed the protective effects of Bak. To summarize, the present study confirmed that Bak can inhibit oxidative stress, attenuate cellular apoptosis, and ameliorate $\mathrm{BBB}$ disruption via regulating the protein level of Trx and the activity of AMPK after SAH (Figure 6).

The pathological mechanisms of EBI include oxidative stress, inflammation, cellular apoptosis, disruption of the BBB, and microvascular dysfunction (Cahill et al., 2006; Sehba et al., 2012). Intercellular contacts between cerebral microvessel endothelial cells participate in the formation of the BBB and are critical to maintaining the brain's structure and function (Keep et al., 2018). These intercellular contacts include tight junctions, the stability of which is maintained by complex networks of occludin, claudin-5, ZO-1 and junctional adhesion molecule (JAM), and adherens junctions, which consist of vascular endothelial (VE) cadherins (Altay et al., 2012; Keep et al., 2018). Proteins in the MMP family are involved in the breakdown of extracellular matrix in the pathological processes of intracerebral hemorrhage (Zhao et al., 2006; Cao et al., 2016). Disruption of the BBB induces vasogenic edema, allows leukocyte extravasation, and allows neurotoxic and vasoactive compounds to leak into the brain (Keep et al., 2018). The BBB disruption and brain edema in EBI after SAH were alleviated by Bak in the present study.

Additionally, the excessive production and release of free radicals, with a weakened scavenger system, disrupt the BBB, leading to brain edema (Park et al., 2004). Reactive oxygen species (ROS) are chemically reactive chemical species containing oxygen including peroxides, superoxide, hydroxyl radical, singlet oxygen, and alpha-oxygen (Hayyan et al., 2016). It is produced in mitochondria by the electron transport chain under physiological conditions. The overload of ROS not only induces oxidative stress damage but also mediates inflammation and apoptosis (Bolanos et al., 2009; Forrester et al., 2018). EBI induces oxidative damage through inhibiting intrinsic antioxidant systems and increasing the production of ROS (Zhang et al., 2016; Liu H. et al., 2017). The production of ROS leads to serious tissue damage by promoting lipid peroxidation, DNA damage, and protein modification (Liu $\mathrm{H}$. et al., 2017). The superoxide anion, as a representative of ROS, was detected by DHE staining to reflect the oxidant stress levels in the tissue in the present study.

Lipid peroxidation is a consequence of free radical-mediated injury in the brain. MDA, 3-NT, and 4-HNE are products of the lipid peroxidation chain reaction and markers which reflect the degree of tissue lipid peroxidation (Gutteridge and Halliwell, 1990; Ayala et al., 2014). 8-OHdG is widely used as a sensitive marker of DNA damage (Di Minno et al., 2016). gp91 ${ }^{\text {phox }}$, a member of the NADPH oxidase (NOX) family, is the primary catalytic subunit of NADPH oxidase which produces reactive oxygen species (Hasegawa et al., 2017). SOD is a member of the 


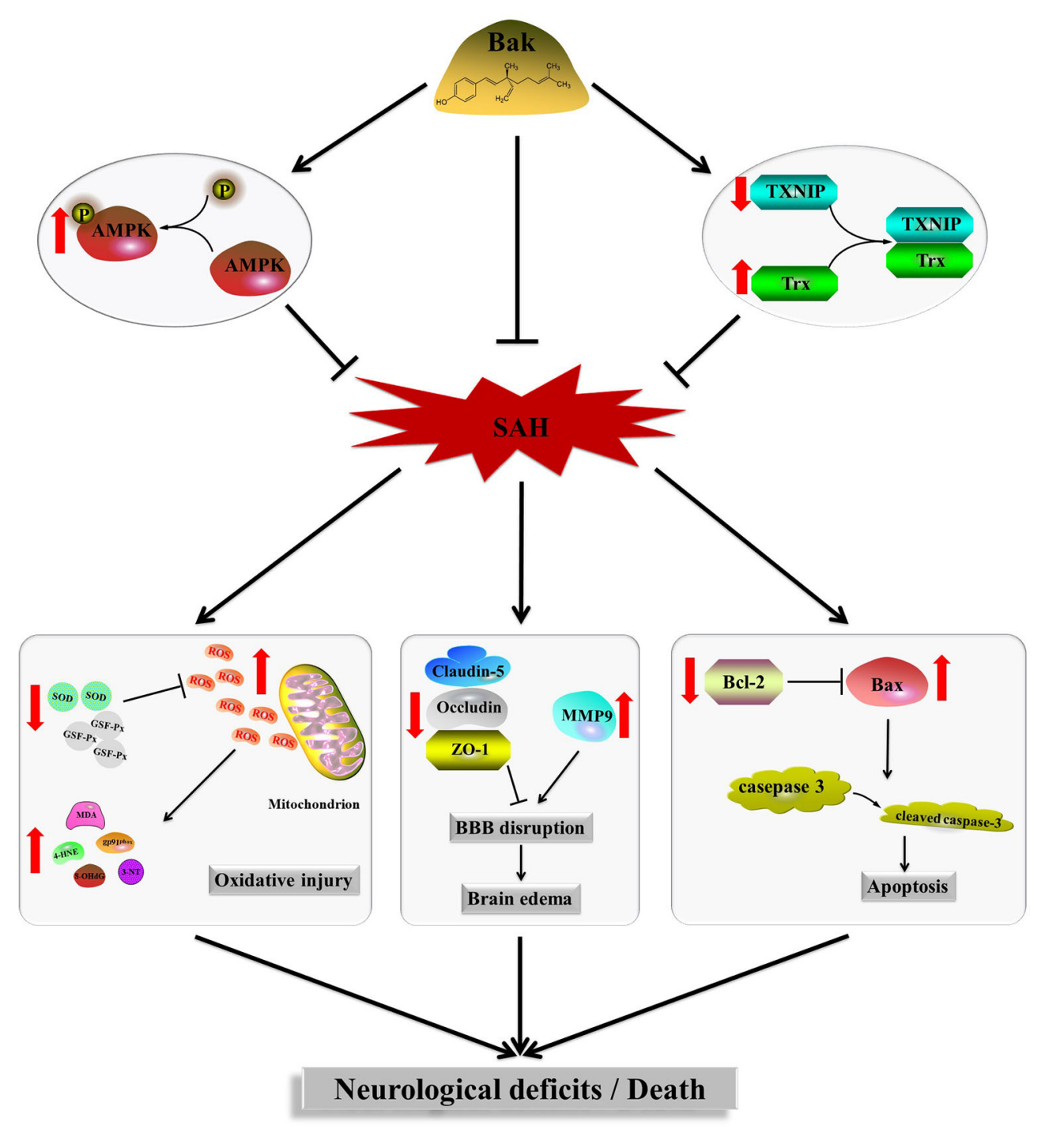

FIGURE 6 | Signaling pathway of Bak's neuroprotective effect against SAH injury which is suggested in the present study. Bak promotes the phosphorylation of AMPK, upregulates the protein level of Trx, and downregulated the protein level of TXNIP, thus alleviates the oxidative stress, cellular apoptosis, brain edema, and neurological deficits after SAH. Bak, bakuchiol; SAH, subarachnoid hemorrhage; Trx, thioredoxin; TXNIP, thioredoxin-interacting protein; AMPK, AMP-activated protein kinase.

enzymatic antioxidative pathway by which the dismutation of superoxide anions into hydrogen peroxide $\left(\mathrm{H}_{2} \mathrm{O}_{2}\right)$ and oxygen $\left(\mathrm{O}_{2}\right)$ is catalyzed. GSH-Px also has notable antioxidative stress effects. Under physiological conditions, SOD, GSH-Px, and other antioxidant molecules comprise the intrinsic antioxidant system and fight against harmful reactive oxygen species ( $\mathrm{Wu}$ et al., 2004; Poprac et al., 2017). The protective effects of Bak are mainly derived from its strong antioxidative properties (Adhikari et al., 2003; Xin et al., 2019). In the present study, Bak simultaneously increased the activities of SOD and GSH-Px and decreased the levels of superoxide production, MDA, 3NT, 8-OHdG, gp91 ${ }^{\text {phox }}$, and 4-HNE in EBI.

ROS can be produced in the endoplasmic reticulum, mitochondria, cytoplasm, and peroxisome. The NOX family is one of the important sources of cytoplasmic ROS, a cornerstone of cellular signaling. Mitochondrial ROS are a natural byproduct of electron transfer in the respiratory chain. Proper respiratory chain function in mitochondria requires a delicate balance between the prooxidant and antioxidant systems (Forrester et al., 2018). In our study, the morphology of mitochondria and endoplasmic reticulum in neurons was observed. Mitochondria were found to be badly impaired, characterized by the loss of mitochondrial cristae, mitochondrial swelling, and collapsed cristae, during EBI, which were notably reversed by Bak administration. The normal morphology of the endoplasmic reticulum was also observed after Bak treatment.

The Trx/TXNIP system has been suggested to be an important contributor to enzymatic systems in ROS generation 
and oxidative stress (Ji et al., 2019). Trx2, the main ROSscavenging enzyme in mitochondria, balances ROS levels and maintains mitochondrial function. Trx1, which is located mainly in the cytoplasm, is usually induced as a response to oxidative stresses (Yoshihara et al., 2014). TXNIP, an endogenous inhibitor of the Trx system, directly binds to Trx1/Trx2 and inhibits their activity through disulfide exchange (Yoshihara et al., 2014; Nasoohi et al., 2018). The inhibition or the deletion of TXNIP is found to be neuroprotective in cerebrovascular and neurodegenerative diseases (Nasoohi et al., 2018). In the present study, the Trx/TXNIP system was involved in the antioxidative mechanism of Bak during EBI.

Cytoplasmic ROS also regulate the activity of AMPK (Hinchy et al., 2018), a central regulator of metabolic functions including lipid metabolism and mitochondrial function (Forrester et al., 2018; Herzig and Shaw, 2018). AMPK is activated by its phosphorylation at threonine-172 and plays a neuroprotective role after SAH (Lin and Hardie, 2018; Enkhjargal et al., 2019). In the present study, an increase in AMPK phosphorylation was observed after Bak administration. Besides, the upregulation of AMPK phosphorylation occurs after SAH in the present study, which has also been reported previously (Osuka et al., 2009; Xu et al., 2019; Xu et al., 2020). In our opinion, this phenomenon may be caused by the compensation mechanism during which the self-protecting signal pathways have been activated to tolerate the stress. In the present study, this mechanism was further enhanced by Bak. More importantly, the protective effects of Bak against BBB disruption, mitochondrial impairment, oxidative stress, and cellular apoptosis were reversed by CC and PX-12.

Cellular apoptosis is the result of oxidative stress-associated lipid peroxidation, protein breakdown, and DNA damage in neurons and endothelial cells during EBI, which leads to neurological deficits (Yuksel et al., 2012; Zhang et al., 2015b). The Bcl-2 family proteins mediate the mitochondrial apoptotic pathway by regulating the release of cytochrome $\mathrm{C}$. The overexpression of $\mathrm{Bcl}-2$ protects cells from apoptosis, whereas the upregulation of Bax reduces the protective effects of Bcl-2 and promotes cell death (Li et al., 2016). We evaluated the expressions of Bcl-2 and Bax and the activity of caspase- 3 after SAH. In the present study, as shown by TUNEL staining and western blotting, Bak protected against apoptosis, which strongly contributed to the alleviation of neurological deficits after SAH.

Previously, it was believed that the apoptosis mainly occurred on neurons after stroke. However, the latest research showed that the apoptosis and cell loss also occurred on glial cells (Chen et al., 2017; Ma et al., 2017; Ning et al., 2017; Sekerdag et al., 2018). Strictly, TUNEL staining alone could not reflect the entire destruction of neurons. However, the FJC staining, detecting the overall neurodegeneration, could reflect the degree of neuron damage more comprehensively. It has been reported that the distribution and degree of FJC-positive cells after SAH were remarkably larger than TUNEL-positive cells (Xie et al., 2015).

The oxidative stress and BBB disruption begin within a few minutes to several hours after stroke and peaks quickly during EBI. By contrast, an important feature of SAH is that there is a delayed phase of brain injury at 3-14 days after hemorrhage in about a third of patients (Macdonald and Schweizer, 2017). The neuronal destruction continues from the EBI phase to the delayed injury phase, resulting in the continuous neurological deterioration (Coulibaly and Provencio, 2020). Considering that neurons are hard to regenerate, this persistent neuronal irreversible structural damage and loss of neurological functions worsen the SAH patients' poor neurological functional outcomes. Therefore, the FJC staining and electron microscopy were conducted at $72 \mathrm{~h}$ after SAH to further evaluate the degree of neuron damage in the present study. Whereas, the long-term changes after 3 days in neuronal structure and neurological functions were not observed, which is a limitation.

With numerous pharmacological properties, Bak exerts a multiorgan protective effect to alleviate damage to the brain (Xin et al., 2019), liver, cardiovascular system, skin, and retina (Kim et al., 2013; Kim S. et al., 2016; Xin et al., 2019). Previous studies have demonstrated that Bak could play antineuroinflammatory effects on activated microglia by the inhibition of the p38 mitogen-activated protein kinases (MAPK)/extracellular signal-regulated kinase 1/2 (ERK) pathways (Lim et al., 2019). ERK could inhibit the activity of AMPK[18]. This evidence is consistent with our findings. Moreover, Bak is proved to be able to suppress lipopolysaccharide (LPS)-stimulated nitric oxide production in LPS-treated BV-2 microglia and shows potent inhibitory activity against hydrogen peroxide-induced cell death in HT22 hippocampal cells (Kim Y. et al., 2016). Bak also has estrogenlike effects (Lim et al., 2011; Weng et al., 2015). Estrogens are strong neuroprotectants that play a protective role in the oxidative stress, mitochondria damage, neuroinflammation, neurodegeneration, and other injury mechanisms in central nervous system (CNS) disease (Miller and Duckles, 2008; Villa et al., 2016; Engler-Chiurazzi et al., 2017). This evidence also suggests that Bak has a strong neuroprotective effect. However, no study about Bak's direct effects on cerebrovascular disease has been reported previously.

In the present study, the protective effects of Bak against $\mathrm{SAH}$ injury have been demonstrated for the first time. The previously demonstrated antioxidative stress and anti-apoptosis effects of Bak have also been further validated in our study. Besides, it has also been confirmed in the present study that AMPK and Trx/ TXNIP play a pivotal role in the antioxidative effect and neuroprotective effect of Bak. The protein kinase activity of AMPK plays a pivotal role in a variety of intracellular biological processes such as the growth of the cell, the metabolism of glucose and lipid, the oxidative stress, the maintenance of mitochondrial function and mitochondrial homeostasis, and the autophagy (Garcia and Shaw, 2017). The Trx system is a main intracellular antioxidant system, which detoxifies ROS and protects cells from oxidative damage (Cheng et al., 2011). While, TXNIP, as an intersection of oxidative stress and inflammasome activation, is essential in both of the oxidative damage and inflammatory injury (Zhou et al., 2010). Therefore, AMPK, Trx, and TXNIP might play a central role in the mechanism of Bak's neuroprotective effect. 
Our findings suggest that Bak has great potential for clinical application in the CNS system. However, the direct molecule target and its particular intracellular signal transduction mechanism of Bak still needs to be further revealed before Bak can be transformed for clinical use. The effects of Bak on other CNS diseases also need to be explored.

The present study was focused on the pretreatment effects of Bak. This route of drug administration is usually used to explore the preventive strategies of diseases. Thus, the findings in the present study can be applied in some clinical occasions. For example, patients are at a high risk of hemorrhage during the neurosurgical procedures dealing with intracranial diseases such as intracranial aneurysms and intracranial arteriovenous malformations. The pretreatment of Bak before surgery might improve patients' tolerance to the possible hemorrhage injury. Besides, periprocedural aneurysm re-rupture is an extremely serious complication of endovascular treatment dealing with aneurysmal SAH. The mortality of patients with aneurysm rerupture is as high as about 70\% (Dmytriw et al., 2014; van Lieshout et al., 2019). The pretreatment of Bak might be a novel preventive therapeutic strategy against the re-rupture of cerebral aneurysms. In addition, patients with unruptured intracranial aneurysms and arteriovenous malformations are at a high risk of stroke when the lesion remains untreated. The preventive taking of Bak could be very beneficial for patients if the stroke occurs. Of course, urgent treatment after SAH is the most difficult problem in clinical practice. However, the effects of Bak posttreatment on SAH injury remain unclear. We will continue to explore this effect in the future.

Limitations still exist in the present study. The direct receptor and signal transduction pathways of Bak have not been fully explored. In subsequent studies, we will continue to explore the upstream and downstream regulators of TRX/TXNIP and AMPK. The effects of Bak on other injury mechanisms after $\mathrm{SAH}$, such as neuroinflammation and autophagy, have not been observed. Thus, there is still a long way to go before the clinical application of Bak.

Another limitation is the measurement method of brain water content. The highest brain water contents in most of the studies using the same animal model did not reach $84 \%$. However, the highest tested brain water content in the present study is about $85 \%$. After careful reflection of our experimental protocol, we believe that the following factors might be the cause of this discrepancy. First, the sutures were advanced approximately 3 more millimeters after feeling the resistance to make sure the vascular was ruptured which might cause more serious traumatic brain injury than the operation in other studies. Second, the

\section{REFERENCES}

Adhikari, S., Joshi, R., Patro, B. S., Ghanty, T. K., Chintalwar, G. J., Sharma, A., et al. (2003). Antioxidant activity of bakuchiol: experimental evidences and theoretical treatments on the possible involvement of the terpenoid chain. Chem. Res. Toxicol. 16 (9), 1062-1069. doi: 10.1021/tx034082r

Altay, O., Suzuki, H., Hasegawa, Y., Caner, B., Krafft, P. R., Fujii, M., et al. (2012). Isoflurane attenuates blood-brain barrier disruption in ipsilateral hemisphere bloodstains and clots were not totally removed from the brain tissue before weighing in the present study. Third, the brains were handled on ice and might be stained with water released from the ice melt. Other unknown factors may also exist, which should be found out in our future study. Besides, the data in the present study cannot be used to compare the degree of cerebral edema at different time points post $\mathrm{SAH}$, which is of great significance in the evolution process of SAH injury.

In conclusion, the present study confirms the protective effects of Bak against EBI after SAH in mice. These effects are mediated through alleviating $\mathrm{BBB}$ impairment, oxidative stress, and apoptosis by regulating Trx/TXNIP and AMPK phosphorylation. The powerful effects of Bak make it a promising novel drug for the treatment of EBI after SAH.

\section{DATA AVAILABILITY STATEMENT}

All datasets generated for this study are included in the article/ supplementary material.

\section{ETHICS STATEMENT}

The animal study was reviewed and approved by Ethics Committee of the Fourth Military Medical University.

\section{AUTHOR CONTRIBUTIONS}

YQ conducted this project and supported the research. HL designed the study and drafted the manuscript. HL, WG, HG, LZ, LY, and XL performed the experiments and acquired the primary data. JL, XW, and WC completed the statistics and interpreted the data. DF revised the manuscript.

\section{FUNDING}

This study was supported by grants from the National Natural Science Foundation of China $(81571215,81630027)$.

\section{ACKNOWLEDGMENTS}

The authors thank the editors from American Journal Experts for their help with professional language editing.

after subarachnoid hemorrhage in mice. Stroke 43 (9), 2513-2516. doi: 10.1161/STROKEAHA.112.661728

Ayala, A., Munoz, M. F., and Arguelles, S. (2014). Lipid peroxidation: production, metabolism, and signaling mechanisms of malondialdehyde and 4-hydroxy-2-nonenal. Oxid. Med. Cell Longev. 2014, 360438. doi: $10.1155 / 2014 / 360438$

Bai, H., Zhao, L., Liu, H., Guo, H., Guo, W., Zheng, L., et al. (2018). Adiponectin confers neuroprotection against cerebral ischemia-reperfusion injury through 
activating the cAMP/PKA-CREB-BDNF signaling. Brain Res. Bull. 143, 145154. doi: 10.1016/j.brainresbull.2018.10.013

Bolanos, J. P., Moro, M. A., Lizasoain, I., and Almeida, A. (2009). Mitochondria and reactive oxygen and nitrogen species in neurological disorders and stroke: Therapeutic implications. Adv. Drug Delivery Rev. 61 (14), 1299-1315. doi: 10.1016/j.addr.2009.05.009

Cahill, J., and Zhang, J. H. (2009). Subarachnoid hemorrhage: is it time for a new direction? Stroke 40 (3 Suppl), S86-S87. doi: 10.1161/STROKEAHA. 108.533315

Cahill, J., Calvert, J. W., and Zhang, J. H. (2006). Mechanisms of early brain injury after subarachnoid hemorrhage. J. Cereb. Blood Flow Metab. 26 (11), 13411353. doi: $10.1038 /$ sj.jcbfm. 9600283

Cao, S., Zhu, P., Yu, X., Chen, J., Li, J., Yan, F., et al. (2016). Hydrogen sulfide attenuates brain edema in early brain injury after subarachnoid hemorrhage in rats: Possible involvement of MMP-9 induced blood-brain barrier disruption and AQP4 expression. Neurosci. Lett. 621, 88-97. doi: 10.1016/ j.neulet.2016.04.018

Chaudhuri, R. K., and Bojanowski, K. (2014). Bakuchiol: a retinol-like functional compound revealed by gene expression profiling and clinically proven to have anti-aging effects. Int. J. Cosmet. Sci. 36 (3), 221-230. doi: 10.1111/ics.12117

Chen, Z., Jin, K., Gao, L., Lou, G., Jin, Y., Yu, Y., et al. (2010). Anti-tumor effects of bakuchiol, an analogue of resveratrol, on human lung adenocarcinoma A549 cell line. Eur. J. Pharmacol. 643 (2-3), 170-179. doi: 10.1016/ j.ejphar.2010.06.025

Chen, X., Lu, M., He, X., Ma, L., Birnbaumer, L., and Liao, Y. (2017). TRPC3/6/7 Knockdown Protects the Brain from Cerebral Ischemia Injury via Astrocyte Apoptosis Inhibition and Effects on NF-small ka, CyrillicB Translocation. Mol. Neurobiol. 54 (10), 7555-7566. doi: 10.1007/s12035-016-0227-2

Cheng, Z., Zhang, J., Ballou, D. P., and Williams, C. H.Jr. (2011). Reactivity of thioredoxin as a protein thiol-disulfide oxidoreductase. Chem. Rev. 111 (9), 5768-5783. doi: $10.1021 / \mathrm{cr} 100006 \mathrm{x}$

Choi, S. Y., Lee, S., Choi, W. H., Lee, Y., Jo, Y. O., and Ha, T. Y. (2010). Isolation and anti-inflammatory activity of Bakuchiol from Ulmus davidiana var. japonica. J. Med. Food 13 (4), 1019-1023. doi: 10.1089/jmf.2009.1207

Coulibaly, A. P., and Provencio, J. J. (2020). Aneurysmal Subarachnoid Hemorrhage: an Overview of Inflammation-Induced Cellular Changes. Neurotherapeutics. doi: 10.1007/s13311-019-00829-x

Di Minno, A., Turnu, L., Porro, B., Squellerio, I., Cavalca, V., Tremoli, E., et al. (2016). 8-Hydroxy-2-Deoxyguanosine Levels and Cardiovascular Disease: A Systematic Review and Meta-Analysis of the Literature. Antioxid. Redox Signal 24 (10), 548-555. doi: 10.1089/ars.2015.6508

Dmytriw, A. A., Pickett, G. E., and Shankar, J. J. (2014). Rupture of aneurysms in the immediate post-coiling period. J. Neurointerv. Surg. 6 (1), 16-18. doi: 10.1136/neurintsurg-2012-010588

Engler-Chiurazzi, E. B., Brown, C. M., Povroznik, J. M., and Simpkins, J. W. (2017). Estrogens as neuroprotectants: Estrogenic actions in the context of cognitive aging and brain injury. Prog. Neurobiol. 157, 188-211. doi: 10.1016/ j.pneurobio.2015.12.008

Enkhjargal, B., Malaguit, J., Ho, W. M., Jiang, W., Wan, W., Wang, G., et al. (2019). Vitamin D attenuates cerebral artery remodeling through VDR/ AMPK/eNOS dimer phosphorylation pathway after subarachnoid hemorrhage in rats. J. Cereb. Blood Flow Metab. 39 (2), 272-284. doi: $10.1177 / 0271678 X 17726287$

Feng, J., Yang, Y., Zhou, Y., Wang, B., Xiong, H., Fan, C., et al. (2016). Bakuchiol attenuates myocardial ischemia reperfusion injury by maintaining mitochondrial function: the role of silent information regulator 1. Apoptosis 21 (5), 532-545. doi: 10.1007/s10495-016-1225-6

Forrester, S. J., Kikuchi, D. S., Hernandes, M. S., Xu, Q., and Griendling, K. K. (2018). Reactive Oxygen Species in Metabolic and Inflammatory Signaling. Circ. Res. 122 (6), 877-902. doi: 10.1161/CIRCRESAHA.117.311401

Fujii, M., Yan, J., Rolland, W. B., Soejima, Y., Caner, B., and Zhang, J. H. (2013). Early brain injury, an evolving frontier in subarachnoid hemorrhage research. Transl. Stroke Res. 4 (4), 432-446. doi: 10.1007/s12975-013-0257-2

Garcia, D., and Shaw, R. J. (2017). AMPK: Mechanisms of Cellular Energy Sensing and Restoration of Metabolic Balance. Mol. Cell 66 (6), 789-800. doi: 10.1016/ j.molcel.2017.05.032

Garcia, J. H., Wagner, S., Liu, K. F., and Hu, X. J. (1995). Neurological deficit and extent of neuronal necrosis attributable to middle cerebral artery occlusion in rats. Statistical validation. Stroke 26 (4), 627-634; discussion 635. doi: 10.1161/ 01.str.26.4.627

Guo, H., Zhao, L., Wang, B., Li, X., Bai, H., Liu, H., et al. (2018). Remote limb ischemic postconditioning protects against cerebral ischemia-reperfusion injury by activating AMPK-dependent autophagy. Brain Res. Bull. 139, 105113. doi: 10.1016/j.brainresbull.2018.02.013

Gutteridge, J. M., and Halliwell, B. (1990). The measurement and mechanism of lipid peroxidation in biological systems. Trends Biochem. Sci. 15 (4), 129-135. doi: 10.1016/0968-0004(90)90206-q

Haraguchi, H., Inoue, J., Tamura, Y., and Mizutani, K. (2000). Inhibition of mitochondrial lipid peroxidation by Bakuchiol, a meroterpene from Psoralea corylifolia. Planta Med. 66 (6), 569-571. doi: 10.1055/s-2000-8605

Hasegawa, Y., Nakagawa, T., Matsui, K., and Kim-Mitsuyama, S. (2017). Renal Denervation in the Acute Phase of Ischemic Stroke Provides Brain Protection in Hypertensive Rats. Stroke 48 (4), 1104-1107. doi: 10.1161/ STROKEAHA.116.015782

Hayyan, M., Hashim, M. A., and AlNashef, I. M. (2016). Superoxide Ion: Generation and Chemical Implications. Chem. Rev. 116 (5), 3029-3085. doi: 10.1021 acs.chemrev.5b00407

Herzig, S., and Shaw, R. J. (2018). AMPK: guardian of metabolism and mitochondrial homeostasis. Nat. Rev. Mol. Cell Biol. 19 (2), 121-135. doi: $10.1038 / \mathrm{nrm} .2017 .95$

Hinchy, E. C., Gruszczyk, A. V., Willows, R., Navaratnam, N., Hall, A. R., Bates, G., et al. (2018). Mitochondria-derived ROS activate AMP-activated protein kinase (AMPK) indirectly. J. Biol. Chem. 293 (44), 17208-17217. doi: 10.1074/ jbc.RA118.002579

Ji, L., Wang, Q., Huang, F., An, T., Guo, F., Zhao, Y., et al. (2019). FOXO1 Overexpression Attenuates Tubulointerstitial Fibrosis and Apoptosis in Diabetic Kidneys by Ameliorating Oxidative Injury via TXNIP-TRX. Oxid. Med. Cell Longev. 2019, 3286928. doi: 10.1155/2019/3286928

Jin, W. N., Shi, S. X., Li, Z., Li, M., Wood, K., Gonzales, R. J., et al. (2017). Depletion of microglia exacerbates postischemic inflammation and brain injury. J. Cereb. Blood Flow Metab. 37 (6), 2224-2236. doi: 10.1177/ 0271678X17694185

Keep, R. F., Andjelkovic, A. V., Xiang, J., Stamatovic, S. M., Antonetti, D. A., Hua, Y., et al. (2018). Brain endothelial cell junctions after cerebral hemorrhage: Changes, mechanisms and therapeutic targets. J. Cereb. Blood Flow Metab. 38 (8), 1255-1275. doi: 10.1177/0271678X18774666

Kim, K. A., Shim, S. H., Ahn, H. R., and Jung, S. H. (2013). Protective effects of the compounds isolated from the seed of Psoralea corylifolia on oxidative stressinduced retinal damage. Toxicol. Appl. Pharmacol. 269 (2), 109-120. doi: 10.1016/j.taap.2013.03.017

Kim, S. J., Oh, H. C., Kim, Y. C., Jeong, G. S., and Lee, S. (2016). Selective Inhibition of Bakuchicin Isolated from Psoralea corylifolia on CYP1A in Human Liver Microsomes. Evid Based Complement Alternat. Med. 2016, 5198743. doi: 10.1155/2016/5198743

Kim, Y. J., Lim, H. S., Lee, J., and Jeong, S. J. (2016). Quantitative Analysis of Psoralea corylifolia Linne and its Neuroprotective and AntiNeuroinflammatory Effects in HT22 Hippocampal Cells and BV-2 Microglia. Molecules 21 (8), E1076. doi: 10.3390/molecules21081076

Kooijman, E., Nijboer, C. H., van Velthoven, C. T., Kavelaars, A., Kesecioglu, J., and Heijnen, C. J. (2014). The rodent endovascular puncture model of subarachnoid hemorrhage: mechanisms of brain damage and therapeutic strategies. J. Neuroinflammation 11, 2. doi: 10.1186/1742-2094-11-2

Krenisky, J. M., Luo, J., Reed, M. J., and Carney, J. R. (1999). Isolation and antihyperglycemic activity of bakuchiol from Otholobium pubescens (Fabaceae), a Peruvian medicinal plant used for the treatment of diabetes. Biol. Pharm. Bull. 22 (10), 1137-1140. doi: 10.1248/bpb.22.1137

Li, J., Chen, J., Mo, H., Chen, J., Qian, C., Yan, F., et al. (2016). Minocycline Protects Against NLRP3 Inflammasome-Induced Inflammation and P53Associated Apoptosis in Early Brain Injury After Subarachnoid Hemorrhage. Mol. Neurobiol. 53 (4), 2668-2678. doi: 10.1007/s12035-015-9318-8

Li, L., Liu, C. C., Chen, X., Xu, S., Hernandez Cortes-Manno, S., and Cheng, S. H. (2017). Mechanistic Study of Bakuchiol-Induced Anti-breast Cancer Stem Cell and in Vivo Anti-metastasis Effects. Front. Pharmacol. 8, 746. doi: 10.3389/ fphar.2017.00746

Li, X., Guo, H., Zhao, L., Wang, B., Liu, H., Yue, L., et al. (2017). Adiponectin attenuates NADPH oxidase-mediated oxidative stress and neuronal damage 
induced by cerebral ischemia-reperfusion injury. Biochim. Biophys. Acta Mol. Basis Dis. 1863 (12), 3265-3276. doi: 10.1016/j.bbadis.2017.08.010

Lim, S. H., Ha, T. Y., Ahn, J., and Kim, S. (2011). Estrogenic activities of Psoralea corylifolia L. seed extracts and main constituents. Phytomedicine 18 (5), 425430. doi: 10.1016/j.phymed.2011.02.002

Lim, H. S., Kim, Y. J., Kim, B. Y., and Jeong, S. J. (2019). Bakuchiol Suppresses Inflammatory Responses Via the Downregulation of the p38 MAPK/ERK Signaling Pathway. Int. J. Mol. Sci. 20 (14), E3574. doi: 10.3390/ijms20143574

Lin, S. C., and Hardie, D. G. (2018). AMPK: Sensing Glucose as well as Cellular Energy Status. Cell Metab. 27 (2), 299-313. doi: 10.1016/j.cmet.2017.10.009

Liu, H., Zhao, L., Yue, L., Wang, B., Li, X., Guo, H., et al. (2017). Pterostilbene Attenuates Early Brain Injury Following Subarachnoid Hemorrhage via Inhibition of the NLRP3 Inflammasome and Nox2-Related Oxidative Stress. Mol. Neurobiol. 54 (8), 5928-5940. doi: 10.1007/s12035-016-0108-8

Liu, L., Kawakita, F., Fujimoto, M., Nakano, F., Imanaka-Yoshida, K., Yoshida, T., et al. (2017). Role of Periostin in Early Brain Injury After Subarachnoid Hemorrhage in Mice. Stroke 48 (4), 1108-1111. doi: 10.1161/ STROKEAHA.117.016629

Lucke-Wold, B. P., Logsdon, A. F., Manoranjan, B., Turner, R. C., McConnell, E., Vates, G. E., et al. (2016). Aneurysmal Subarachnoid Hemorrhage and Neuroinflammation: A Comprehensive Review. Int. J. Mol. Sci. 17 (4), 497. doi: 10.3390/ijms17040497

Ma, Y. L., Zhang, L. X., Liu, G. L., Fan, Y., Peng, Y., and Hou, W. G. (2017). N-Myc Downstream-Regulated Gene 2 (Ndrg2) Is Involved in Ischemia-HypoxiaInduced Astrocyte Apoptosis: a Novel Target for Stroke Therapy. Mol. Neurobiol. 54 (5), 3286-3299. doi: 10.1007/s12035-016-9814-5

Macdonald, R. L., and Schweizer, T. A. (2017). Spontaneous subarachnoid haemorrhage. Lancet 389 (10069), 655-666. doi: 10.1016/S0140-6736(16) 30668-7

Macdonald, R. L. (2014). Delayed neurological deterioration after subarachnoid haemorrhage. Nat. Rev. Neurol. 10 (1), 44-58. doi: 10.1038/nrneurol.2013.246

Miller, V. M., and Duckles, S. P. (2008). Vascular actions of estrogens: functional implications. Pharmacol. Rev. 60 (2), 210-241. doi: 10.1124/pr.107.08002

Nasoohi, S., Ismael, S., and Ishrat, T. (2018). Thioredoxin-Interacting Protein (TXNIP) in Cerebrovascular and Neurodegenerative Diseases: Regulation and Implication. Mol. Neurobiol. 55 (10), 7900-7920. doi: 10.1007/s12035-018-0917-z

Ning, B., Guo, G., Liu, H., Ning, L., Sun, B. L., Li, Z., et al. (2017). MSK1 downregulation is associated with neuronal and astrocytic apoptosis following subarachnoid hemorrhage in rats. Oncol. Lett. 14 (3), 2940-2946. doi: 10.3892/ ol.2017.6496

Oliveira, S. M., Zhang, Y. H., Solis, R. S., Isackson, H., Bellahcene, M., Yavari, A., et al. (2012). AMP-activated protein kinase phosphorylates cardiac troponin I and alters contractility of murine ventricular myocytes. Circ. Res. 110 (9), 1192-1201. doi: 10.1161/CIRCRESAHA.111.259952

Osuka, K., Watanabe, Y., Usuda, N., Atsuzawa, K., Yoshida, J., and Takayasu, M. (2009). Modification of endothelial nitric oxide synthase through AMPK after experimental subarachnoid hemorrhage. J. Neurotrauma 26 (7), 1157-1165. doi: 10.1089/neu.2008-0836

Park, S., Yamaguchi, M., Zhou, C., Calvert, J. W., Tang, J., and Zhang, J. H. (2004). Neurovascular protection reduces early brain injury after subarachnoid hemorrhage. Stroke 35 (10), 2412-2417. doi: 10.1161/01.STR.0000141162. 29864.e9

Poprac, P., Jomova, K., Simunkova, M., Kollar, V., Rhodes, C. J., and Valko, M. (2017). Targeting Free Radicals in Oxidative Stress-Related Human Diseases. Trends Pharmacol. Sci. 38 (7), 592-607. doi: 10.1016/j.tips.2017.04.005

Sehba, F. A., Hou, J., Pluta, R. M., and Zhang, J. H. (2012). The importance of early brain injury after subarachnoid hemorrhage. Prog. Neurobiol. 97 (1), 14-37. doi: 10.1016/j.pneurobio.2012.02.003

Sekerdag, E., Solaroglu, I., and Gursoy-Ozdemir, Y. (2018). Cell Death Mechanisms in Stroke and Novel Molecular and Cellular Treatment Options. Curr. Neuropharmacol. 16 (9), 1396-1415. doi: 10.2174/1570159X 16666180302115544

Seo, E., Oh, Y. S., Kim, D., Lee, M. Y., Chae, S., and Jun, H. S. (2013). Protective Role of Psoralea corylifolia L. Seed Extract against Hepatic Mitochondrial Dysfunction Induced by Oxidative Stress or Aging. Evid Based Complement Alternat. Med. 2013, 678028. doi: 10.1155/2013/678028

Sozen, T., Tsuchiyama, R., Hasegawa, Y., Suzuki, H., Jadhav, V., Nishizawa, S., et al. (2009). Role of interleukin-1beta in early brain injury after subarachnoid hemorrhage in mice. Stroke 40 (7), 2519-2525. doi: 10.1161/STROKEAHA. 109.549592

Sugawara, T., Ayer, R., Jadhav, V., and Zhang, J. H. (2008). A new grading system evaluating bleeding scale in filament perforation subarachnoid hemorrhage rat model. J. Neurosci. Methods 167 (2), 327-334. doi: 10.1016/j.jneumeth. 2007.08.004

van Gijn, J., Kerr, R. S., and Rinkel, G. J. (2007). Subarachnoid haemorrhage. Lancet 369 (9558), 306-318. doi: 10.1016/S0140-6736(07)60153-6

van Lieshout, J. H., Verbaan, D., Donders, R., van den Berg, R., Vandertop, P. W. P., Klijn, C. J. M., et al. (2019). Periprocedural aneurysm rerupture in relation to timing of endovascular treatment and outcome. J. Neurol. Neurosurg. Psychiatry 90 (3), 363-365. doi: 10.1136/jnnp-2018-318090

Villa, A., Vegeto, E., Poletti, A., and Maggi, A. (2016). Estrogens, Neuroinflammation, and Neurodegeneration. Endocr. Rev. 37 (4), 372-402. doi: 10.1210/er.2016-1007

Weng, Z. B., Gao, Q. Q., Wang, F., Zhao, G. H., Yin, F. Z., Cai, B. C., et al. (2015). Positive skeletal effect of two ingredients of Psoralea corylifolia L. @ on estrogen deficiency-induced osteoporosis and the possible mechanisms of action. Mol. Cell Endocrinol. 417, 103-113. doi: 10.1016/j.mce.2015.09.025

Wu, L., Noyan Ashraf, M. H., Facci, M., Wang, R., Paterson, P. G., Ferrie, A., et al. (2004). Dietary approach to attenuate oxidative stress, hypertension, and inflammation in the cardiovascular system. Proc. Natl. Acad. Sci. U. S. A 101 (18), 7094-7099. doi: 10.1073/pnas.0402004101

Xi, G., Hua, Y., Bhasin, R. R., Ennis, S. R., Keep, R. F., and Hoff, J. T. (2001). Mechanisms of edema formation after intracerebral hemorrhage: effects of extravasated red blood cells on blood flow and blood-brain barrier integrity. Stroke 32 (12), 2932-2938. doi: 10.1161/hs1201.099820

Xie, Y., Liu, W., Zhang, X., Wang, L., Xu, L., Xiong, Y., et al. (2015). Human Albumin Improves Long-Term Behavioral Sequelae After Subarachnoid Hemorrhage Through Neurovascular Remodeling. Crit. Care Med. 43 (10), e440-e449. doi: 10.1097/CCM.0000000000001193

Xin, Z., Wu, X., Ji, T., Xu, B., Han, Y., Sun, M., et al. (2019). Bakuchiol: A newly discovered warrior against organ damage. Pharmacol. Res. 141, 208-213. doi: 10.1016/j.phrs.2019.01.001

Xu, W., Li, T., Gao, L., Zheng, J., Yan, J., Zhang, J., et al. (2019). Apelin-13/APJ system attenuates early brain injury via suppression of endoplasmic reticulum stress-associated TXNIP/NLRP3 inflammasome activation and oxidative stress in a AMPK-dependent manner after subarachnoid hemorrhage in rats. J. Neuroinflammation 16 (1), 247. doi: 10.1186/s12974-019-1620-3

Xu, W., Mo, J., Ocak, U., Travis, Z. D., Enkhjargal, B., Zhang, T., et al. (2020). Activation of Melanocortin 1 Receptor Attenuates Early Brain Injury in a Rat Model of Subarachnoid Hemorrhage viathe Suppression of Neuroinflammation through AMPK/TBK1/NF-kappaB Pathway in Rats. Neurotherapeutics 17 (1), 294-308. doi: 10.1007/s13311-019-00772-x

Yoshihara, E., Masaki, S., Matsuo, Y., Chen, Z., Tian, H., and Yodoi, J. (2014). Thioredoxin/Txnip: redoxisome, as a redox switch for the pathogenesis of diseases. Front. Immunol. 4, 514. doi: 10.3389/fimmu.2013.00514

Yuksel, S., Tosun, Y. B., Cahill, J., and Solaroglu, I. (2012). Early brain injury following aneurysmal subarachnoid hemorrhage: emphasis on cellular apoptosis. Turk Neurosurg. 22 (5), 529-533. doi: 10.5137/1019-5149.JTN.5731-12.1

Zhang, X. S., Zhang, X., Zhou, M. L., Zhou, X. M., Li, N., Li, W., et al. (2014). Amelioration of oxidative stress and protection against early brain injury by astaxanthin after experimental subarachnoid hemorrhage. J. Neurosurg. 121 (1), 42-54. doi: 10.3171/2014.2.JNS13730

Zhang, Z. Y., Sun, B. L., Yang, M. F., Li, D. W., Fang, J., and Zhang, S. (2015a). Carnosine attenuates early brain injury through its antioxidative and antiapoptotic effects in a rat experimental subarachnoid hemorrhage model. Cell Mol. Neurobiol. 35 (2), 147-157. doi: 10.1007/s10571-014-0106-1

Zhang, Z. Y., Yang, M. F., Wang, T., Li, D. W., Liu, Y. L., Zhang, J. H., et al. (2015b). Cysteamine alleviates early brain injury via reducing oxidative stress and apoptosis in a rat experimental subarachnoid hemorrhage model. Cell Mol. Neurobiol. 35 (4), 543-553. doi: 10.1007/s10571-014-0150-x

Zhang, L., Wu, J., Duan, X., Tian, X., Shen, H., Sun, Q., et al. (2016). NADPH Oxidase: A Potential Target for Treatment of Stroke. Oxid. Med. Cell Longev. 2016, 5026984. doi: 10.1155/2016/5026984

Zhang, X., Chang, N., Zhang, Y., Ye, M., Han, Z., Li, J., et al. (2017). Bakuchiol Protects Against Acute Lung Injury in Septic Mice. Inflammation 40 (2), 351359. doi: $10.1007 / \mathrm{s} 10753-016-0481-5$ 
Zhao, B. Q., Wang, S., Kim, H. Y., Storrie, H., Rosen, B. R., Mooney, D. J., et al. (2006). Role of matrix metalloproteinases in delayed cortical responses after stroke. Nat. Med. 12 (4), 441-445. doi: 10.1038/nm1387

Zhao, L., Liu, H., Yue, L., Zhang, J., Li, X., Wang, B., et al. (2017). Melatonin Attenuates Early Brain Injury via the Melatonin Receptor/Sirt1/NF-kappaB Signaling Pathway Following Subarachnoid Hemorrhage in Mice. Mol. Neurobiol. 54 (3), 1612-1621. doi: 10.1007/s12035-016-9776-7

Zhou, R., Tardivel, A., Thorens, B., Choi, I., and Tschopp, J. (2010). Thioredoxininteracting protein links oxidative stress to inflammasome activation. Nat. Immunol. 11 (2), 136-140. doi: 10.1038/ni.1831
Conflict of Interest: The authors declare that the research was conducted in the absence of any commercial or financial relationships that could be construed as a potential conflict of interest.

Copyright (C) $2020 \mathrm{Liu}, \mathrm{Guo}, \mathrm{Guo}, \mathrm{Zhao}$, Yue, Li, Feng, Luo, Wu, Cui and Qu. This is an open-access article distributed under the terms of the Creative Commons Attribution License (CC BY). The use, distribution or reproduction in other forums is permitted, provided the original author(s) and the copyright owner(s) are credited and that the original publication in this journal is cited, in accordance with accepted academic practice. No use, distribution or reproduction is permitted which does not comply with these terms. 